\title{
The formin DAAM is required for coordination of the actin and microtubule cytoskeleton in axonal growth cones
}

\author{
Szilárd Szikora1, István Földi ${ }^{1}$, Krisztina Tóth ${ }^{1}$, Ede Migh $^{1}$, Andrea Vig $^{2}$, Beáta Bugyi ${ }^{2,3}$, József Maléth ${ }^{4}$, \\ Péter Hegyi ${ }^{4,5}$, Péter Kaltenecker ${ }^{6}$, Natalia Sanchez-Soriano ${ }^{6}$ and József Mihály ${ }^{1, *}$
}

\begin{abstract}
Directed axonal growth depends on correct coordination of the actin and microtubule cytoskeleton in the growth cone. However, despite the relatively large number of proteins implicated in actin-microtubule crosstalk, the mechanisms whereby actin polymerization is coupled to microtubule stabilization and advancement in the peripheral growth cone remained largely unclear. Here, we identified the formin Dishevelled-associated activator of morphogenesis (DAAM) as a novel factor playing a role in concerted regulation of actin and microtubule remodeling in Drosophila melanogaster primary neurons. In vitro, DAAM binds to F-actin as well as to microtubules and has the ability to crosslink the two filament systems. Accordingly, DAAM associates with the neuronal cytoskeleton, and a significant fraction of DAAM accumulates at places where the actin filaments overlap with that of microtubules. Loss of DAAM affects growth cone and microtubule morphology, and several aspects of microtubule dynamics; and biochemical and cellular assays revealed a microtubule stabilization activity and binding to the microtubule tip protein EB1. Together, these data suggest that, besides operating as an actin assembly factor, DAAM is involved in linking actin remodeling in filopodia to microtubule stabilization during axonal growth.
\end{abstract}

KEY WORDS: Actin, Microtubule, Formin, DAAM, Growth cone, Drosophila

\section{INTRODUCTION}

Directional movement of axons, governed by their distally located growth cones, is elicited by coordinated changes of peripheral F-actin (filamentous-actin) and central microtubule networks (Dent and Gertler, 2003; Lowery and Van Vactor, 2009). F-actin in growth cone periphery is localized to two main structures, filopodia and lamellipodia. Splayed microtubules emanating from the central axonal microtubule bundles dynamically invade and scan the F-actin-rich periphery, often reaching deep into filopodia (Dent and Gertler, 2003; Lowery and Van Vactor, 2009). Filopodial F-actin bundles can act as guides for these dynamic microtubules, and it is thought that this actin-microtubule crosstalk followed by

\footnotetext{
${ }^{1}$ Institute of Genetics, Biological Research Centre, Hungarian Academy of Sciences, MTA-SZBK NAP B Axon Growth and Regeneration Group, Temesvári krt.

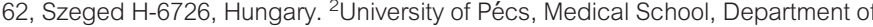
Biophysics, Szigeti str. 12, Pécs H-7624, Hungary. ${ }^{3}$ Szentágothai Research Center, Ifjúság str. 34, Pécs H-7624, Hungary. ${ }^{4}$ MTA-SZTE Translational Gastroenterology Research Group, First Department of Internal Medicine, Szeged H-6720, Hungary. ${ }^{5}$ Institute for Translational Medicine, Department of Pathophysiology, University of Pécs, Pécs H-7624, Hungary. ${ }^{6}$ Institute for Translational Medicine, Department of Cellular and Molecular Physiology, University of Liverpool, Liverpool L69 3BX, UK.

*Author for correspondence (mihaly.jozsef@brc.mta.hu)
}

(D) K.T., 0000-0002-2506-7127; J.M., 0000-0003-3399-2424

Received 2 March 2017; Accepted 5 June 2017 microtubule and filopodia stabilization is a key step of growth cone advance. Most proteins that have so far been linked to the molecular mechanisms of interaction between actin and microtubules belong to the microtubule plus-end tracking proteins (+TIPs), such as CLASP, CLIP-170 (also known as CLIP1), APC, EB1 and EB3 (also known as MAPRE1 and MAPRE3, respectively), LIS1 (also known as PAFAH1B1) and the spectraplakin ACF7 (also known as MACF1) (recently reviewed in Bearce et al., 2015; Cammarata et al., 2016; Coles and Bradke, 2015). Although the role of these proteins in growth cone guidance is supported by genetic, biochemical and advanced microscopic assays, these large proteins operate in complex interaction networks posing a great challenge for dissecting how they precisely function.

Beyond the classic +TIPs, the number of proteins that may couple microtubules to F-actin dynamics at plus-ends is still expanding (Coles and Bradke, 2015; Jiang et al., 2012). For example, recent work identified members of the formin family as regulators of crosstalk between actin and microtubules in non-neuronal cells (Bartolini et al., 2008; Chesarone et al., 2010; Gaillard et al., 2011; Rosales-Nieves et al., 2006; Young and Copeland, 2010). Formins are well-characterized actin assembly factors that promote the formation of unbranched actin cables by facilitating their nucleation and elongation (Chesarone et al., 2010; Schönichen and Geyer, 2010). These multidomain proteins contain two highly conserved signature domains, the formin homology 1 and 2 (FH1 and FH2, respectively) domains. The dimeric FH2 nucleates actin filaments and supports elongation by remaining attached to their barbed ends (Higashida et al., 2004; Kovar and Pollard, 2004; Watanabe and Higashida, 2004), whereas the proline-rich FH1 domain accelerates elongation by recruiting profilin-bound actin monomers ( $\mathrm{Li}$ and Higgs, 2003; Sagot et al., 2002). Some formins contain several other conserved regions including the N-terminally localized GTPasebinding domain (GBD), diaphanous inhibitory domain (DID), dimerization domain (DD), Coiled-coil region $(\mathrm{CC})$ and the $\mathrm{C}$ terminal diaphanous auto-regulatory domain (DAD) (Alberts, 2001; Chesarone et al., 2010; Li and Higgs, 2003; Otomo et al., 2005; Rose et al., 2005). These domains are involved in the spatial and temporal regulation of the actin assembly activity provided by the FH2 domain (Chesarone et al., 2010). In addition, it has also been shown that a number of formins influence microtubule (MT) stability and organization (Bartolini and Gundersen, 2010). Consistently, many formins are able to bind to MTs in vitro through their actin-binding FH2 domain and in some cases with their C-terminal region (Bartolini et al., 2008; Chesarone et al., 2010; Gaillard et al., 2011; Roth-Johnson et al., 2014; Young et al., 2008; Zhou et al., 2006). The presence of both an actin- and a microtubule-binding domain might allow these proteins to crosslink the cytoskeletal components directly, such as Cappuccino does in vitro - and presumably in vivo - in Drosophila oocytes (RosalesNieves et al., 2006). By contrast, instead of - or in parallel to - 
direct binding, the Diaphanous-related formins (DRFs) mDia1, $\mathrm{mDia} 2$ and $\mathrm{mDia} 3$ appear to stabilize MTs in fibroblast cells by binding +TIP through EB1, CLIP-170 or APC (Cheng et al., 2011; Lewkowicz et al., 2008; Wen et al., 2004). Interestingly, mDia2 is able to stabilize MTs and bind to +TIPs independently of its actin nucleation activity (Bartolini et al., 2008). Finally, recent work revealed that CLIP-170 binds tightly to mDial to increase the rate of actin polymerization from MT plus-ends (Henty-Ridilla et al., 2016). Despite these advances, the in vivo contribution of formins to MT and F-actin interactions in axonal growth cones remained elusive.

In this paper, we characterize the role of the formin family protein Dishevelled-associated activator of morphogenesis (DAAM) in concerted regulation of actin and MT remodeling within axons of Drosophila neurons. We show that Drosophila DAAM partially colocalizes with both main cytoskeletal systems in neurons, and it is able to crosslink actin filaments with MTs in vitro. DAAM stabilizes MTs against cold- or nocodazole-induced depolymerization, and the absence of $D A A M$ impairs neuronal MT organization and dynamics. Although DAAM has the ability to bind MTs directly, it also binds to the MT plus-end tracking protein EB1, and often localizes to MT plus-tips. This suggests that, similarly to the process in migrating cells, a formin-/+TIP-dependent mechanism is crucial to govern actin and MT coordination in growing axons.

\section{RESULTS \\ DAAM localizes to axonal microtubule bundles and physically interacts with microtubules}

Previously, we have shown that DAAM plays an essential role in the differentiation of the embryonic nervous system (Matusek et al., 2008) and the adult brain (Gombos et al., 2015). In vitro and in vivo studies revealed that DAAM behaves as a bona fide formin, and it is required for filopodia formation (Barko et al., 2010; Matusek et al., 2008) like its vertebrate ortholog Daaml (Jaiswal et al., 2013a). Furthermore, the observed cellular phenotypes, i.e. reduced number of filopodia and reduced length coincide with DAAM accumulation along the shaft and on the tip of filopodia (Gonçalves-Pimentel et al., 2011; Jaiswal et al., 2013a; Matusek et al., 2008). Although formins are primarily considered as actin assembly machineries, numerous studies have demonstrated that some formins are able to interact with MTs either directly or indirectly (Bartolini and Gundersen, 2010; Bartolini et al., 2008; Dahlgaard et al., 2007; Gaillard et al., 2011; Gasteier et al., 2005; Rosales-Nieves et al., 2006; Roth-Johnson et al., 2014; Shaye and Greenwald, 2015). Since the highly conserved FH2 was identified as the main MTinteracting domain in DRFs, it is reasonable to assume that DAAM is also able to interact with MTs. Therefore, to further elaborate on the functions of DAAM, we reassessed its sub-cellular localization by performing immunostaining of endogenous DAAM in cultured primary neurons. We found that, although DAAM primarily localizes to F-actin-rich structures, i.e. to filopodia, lamellipodia and cortical actin (Fig. S1A-D,I,I'; Movie 1), a significant fraction of DAAM accumulated in punctae along the axonal MTs (Fig. 1A-D, Fig. S1E-H). To estimate the sub-cellular distribution of DAAM, we quantified the protein-protein proximity index (PPI) (Wu et al., 2010; Zinchuk et al., 2011) between DAAM and actin, and DAAM and tubulin, respectively. The analysis revealed that $\sim 47 \%$ (PPI: $0.47 \pm 0.05$ ) of DAAM is associated with the actin cytoskeleton. Surprisingly, however, another $\sim 37 \%$ (PPI: $0.37 \pm$ 0.05) exhibited an overlap with MTs (Fig. 1E). Most notably, we detected frequent DAAM accumulation on axonal MT ends $(36 \pm 14 \%)$ (Fig. 1F). These observations were further verified with a second, independently created DAAM antibody (Fig. S3G), leading to very similar results (Fig. S1E-H). Thus, besides actin, DAAM is often localized to MTs in axonal growth cones pointing towards MT-related functions.

To begin to address whether DAAM interacts with MTs, GSTtagged DAAM fragments were purified and, subsequently, used in MT co-sedimentation and GST pull-down assays to test for direct interaction (Fig. 1G,H and Fig. S2A-E). Both assays indicated that, unlike the purified GST protein used as negative control, a DAAM fragment comprising the $\mathrm{FH} 1$ and $\mathrm{FH} 2$ domains was able to bind to taxol-stabilized MTs, whereas mutation of the conserved amino acid isoleucine (I732A) that plays a crucial role in actin interaction did not affect interaction between GST::FH1FH2 and MTs. In addition to the $\mathrm{FH} 2$ domain, the $\mathrm{C}$-terminal region of some formins was also shown to contribute to MT binding (Gaillard et al., 2011; RothJohnson et al., 2014; Young et al., 2008); thus, we also investigated the effect of the C-terminal region of DAAM. It appeared that the DAD domain alone did not bind to MTs but that the C-terminal tail (CT) region has MT-binding capability. It has been shown that MT binding through the tail region of different formins is mediated by ionic interactions between basic amino acids of the tail domain and the acidic MT surface (Gaillard et al., 2011; Roth-Johnson et al., 2014). The CT domain of DAAM is also basic with a pI of 11.6. and, in line with previous data, a mutant CT fragment (GST::DAD-CT $\mathrm{T}^{\mathrm{R}-\mathrm{A}}$ ) in which the basic amino acids had been changed to alanines was not able to bind to taxol-stabilized MTs.

In addition to direct MT binding, several formins were shown to associate with MTs through + TIP binding. Consistent with its MT plus-end localization (Fig. 1F), DAAM was co-immunoprecipitated with EB1 (an abundant +TIP in this system) from lysates of S2 cells transfected with $3 \times$ FLAG-tagged constitutively active DAAM lacking the DAD domain (Fig. 1I). Collectively, these studies indicate that Drosophila DAAM, similarly to other formins, has the ability to physically interact with MTs via its FH2 as well as CT domains. Besides direct interaction, DAAM co-purifies with EB1 suggesting an alternative mode of MT binding, presumably restricted to MT plus-ends.

\section{DAAM regulates the length and organization of axonal microtubule bundles}

The sub-cellular localization and the in vitro assays suggest that DAAM plays a role in the regulation of MT organization during axon growth. Therefore, we next asked how loss of DAAM affects MT morphology in primary neurons. Since the maternal $D A A M$ product is present in the embryos (Matusek et al., 2006), we generated primary neuronal cultures from $D A A M^{\text {mattzyg }}$ mutant embryos (Matusek et al., 2008 ) in which maternal and zygotic DAAM functions are both impaired. Previously, we have demonstrated that these neurons are able to develop axons similar to the wild-type control neurons, although the number and length of growth cone filopodia are significantly decreased (Matusek et al., 2008). In this present study we measured the length of the axonal MT bundles after tubulin staining by using the semi-automatic NeuronJ plugin (Meijering et al., 2004) and revealed that axonal MT bundles and, consequently, axons are significantly $(P=0.016)$ shorter in the $D A A M^{\text {mat } z y g}$ mutant neurons $(9.67 \pm 0.75 \mu \mathrm{m}$, $n=152)$ as compared to wild-type controls $(16.36 \pm 2.2 \mu \mathrm{m}, n=235)$ (Fig. 2A-B' and F). Moreover, we also studied MT morphology (Fig. 2E and G), and noticed that, in $D A A M^{\text {mat/zyg }}$ mutant neurons, the frequency of looped and spread MTs is decreased, while the frequency of bundled MTs is increased significantly $(P=0.0286)$ (Fig. $2 \mathrm{G})$.

To further elaborate on the role of DAAM, we overexpressed an activated form of DAAM (CDAAM) that lacks its N-terminal 

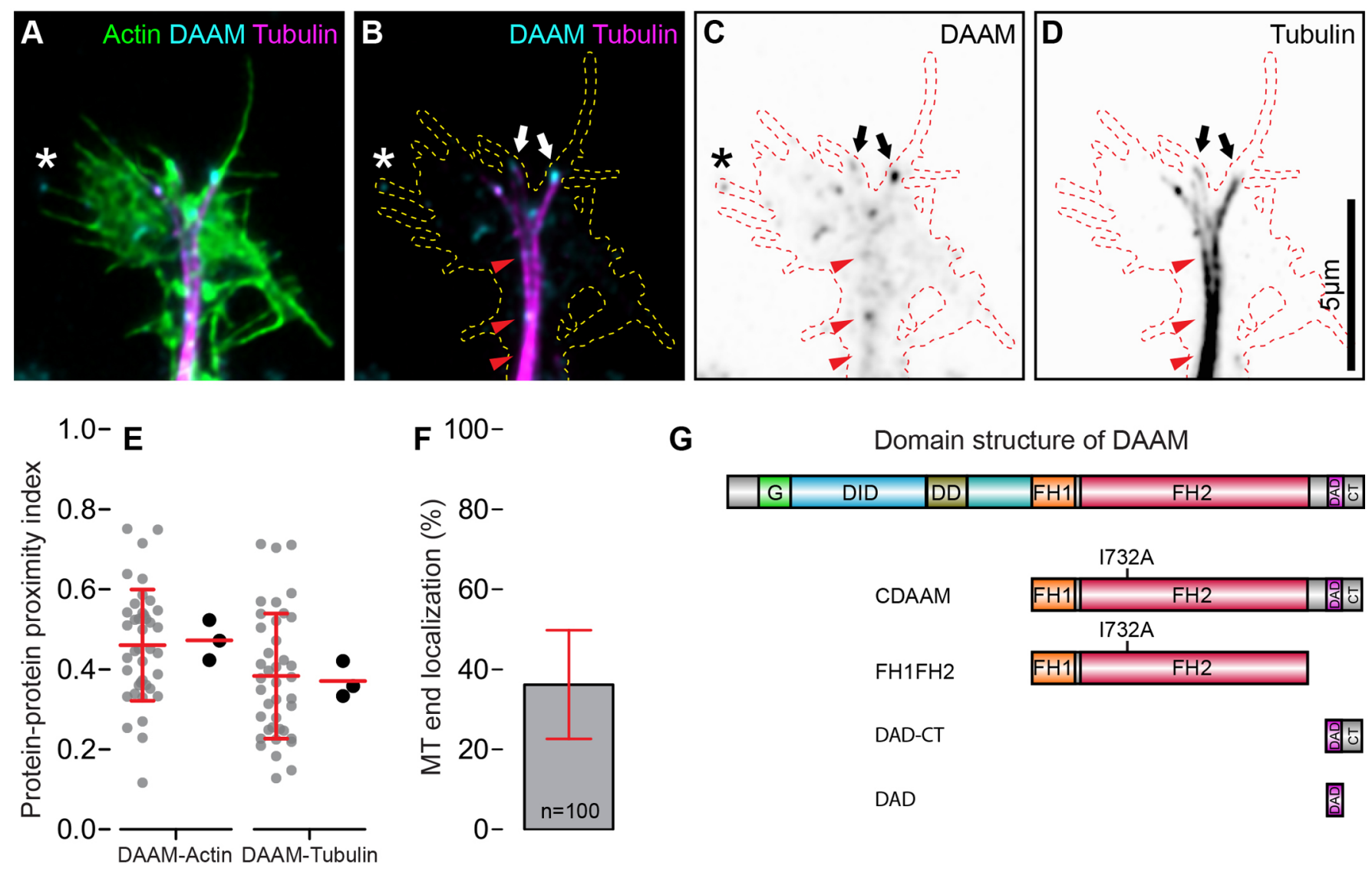

$\mathbf{F}^{100-}$

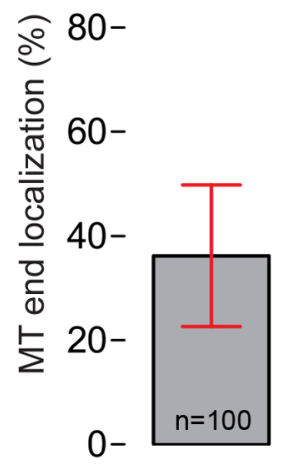

G

Domain structure of DAAM

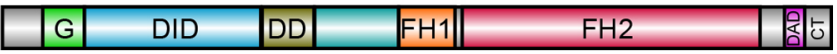

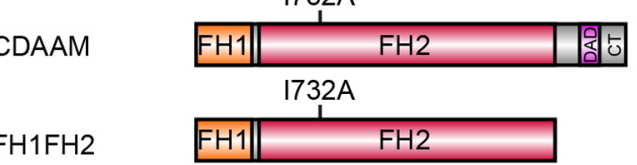

DAD-CT

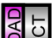

\section{H}
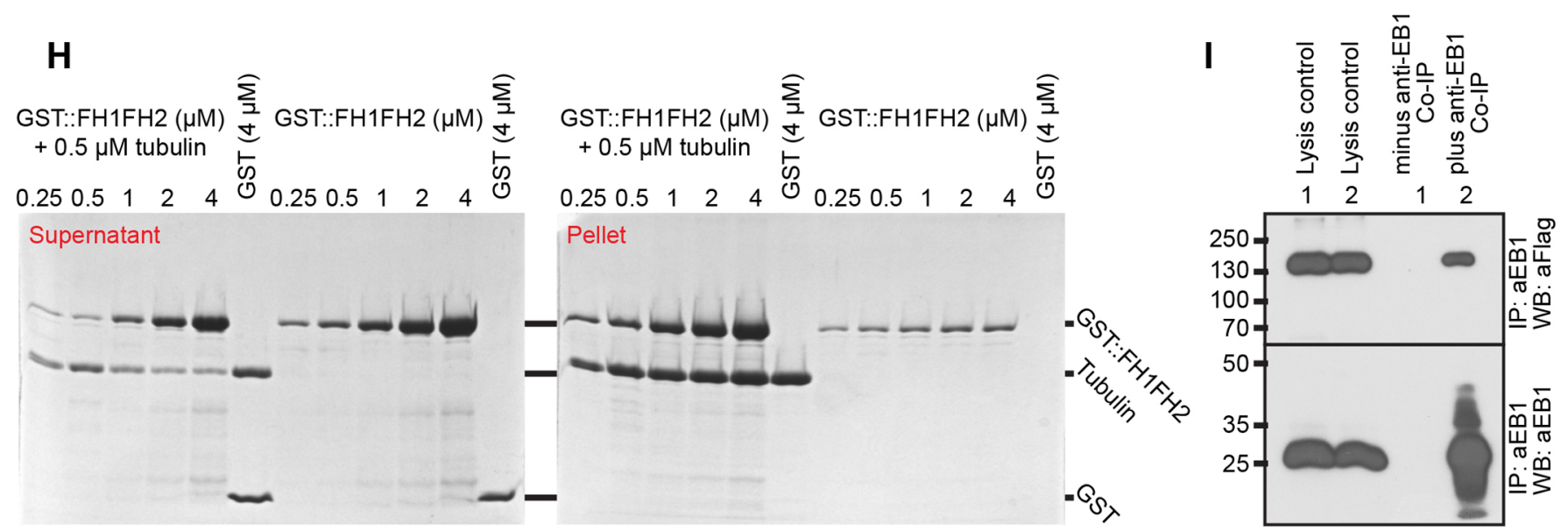

Fig. 1. Axonal localization and physical interaction of DAAM with microtubules. (A-D) Growth cone of a primary neuron (6 HIV), labeled against F-actin, tubulin and DAAM. Asterisk marks filopodial DAAM localization. Arrows point to DAAM puncta localized at the end of microtubules. Arrowheads show DAAM localization along the MT bundles. (E) Scatterplots show the protein-protein proximity index measured between DAAM and F-actin, and DAAM and tubulin. Gray dots represent values measured on individual cells (DAAM-Actin, 0.46 \pm 0.14 , mean $\pm s . d$. $n=40 ; D A A M-T u b u l i n, 0.38 \pm 0.15$, mean $\pm s$.d. $n=40$ ). Black dots represent the median of the independent experiments with their median. (F) Bar diagram shows the frequency of microtubule end localization of DAAM (36.18\% \pm $13.57 \%$, mean \pm s.d.; measured on 100 MT ends from three independent experiments). (G) Domain structure of full-length DAAM and DAAM fragments used in this study. (H) MT co-sedimentation assay shows that MTs physically interact with the FH1FH2 fragment of dDAAM. (I) Co-immunoprecipitation shows that $\triangle \mathrm{DAD}-\mathrm{DAAM}:: 3 \times \mathrm{FLAG}$ co-precipitates specifically with EB1 from S2 cell lysates.

regions including the DID domain - which is crucial for autoinhibition (Liu et al., 2008; Li and Higgs, 2005) - by using the pan-neuronal elav-Gal4 driver. Similarly to $D A A M^{\text {mat/zyg }}$ mutant neurons, the axonal MT bundles were shorter $(P=0.0011)$ in CDAAM-expressing cells $(14.88 \pm 0.21 \mu \mathrm{m}, n=178)$ as compared to controls $(21.51 \pm 1.15 \mu \mathrm{m}, n=182)$ (Fig. $2 \mathrm{C}-\mathrm{D}^{\prime}$ and F). In contrast to $D A A M^{\text {mat } / z y g}$ neurons, the frequency of looped and spread MTs increased, while the frequency of bundled MTs decreased in
CDAAM-expressing cells (Fig. 2G). Thus, the loss- and gain-offunction studies both indicate a role in the regulation of axonal microtubule length and organization.

\section{DAAM regulates axonal microtubule dynamics}

As deviations in the organization of axonal MTs are often linked to impaired MT dynamics, we next sought to examine MT dynamics in vivo, in the absence of DAAM. Microtubule dynamics were 

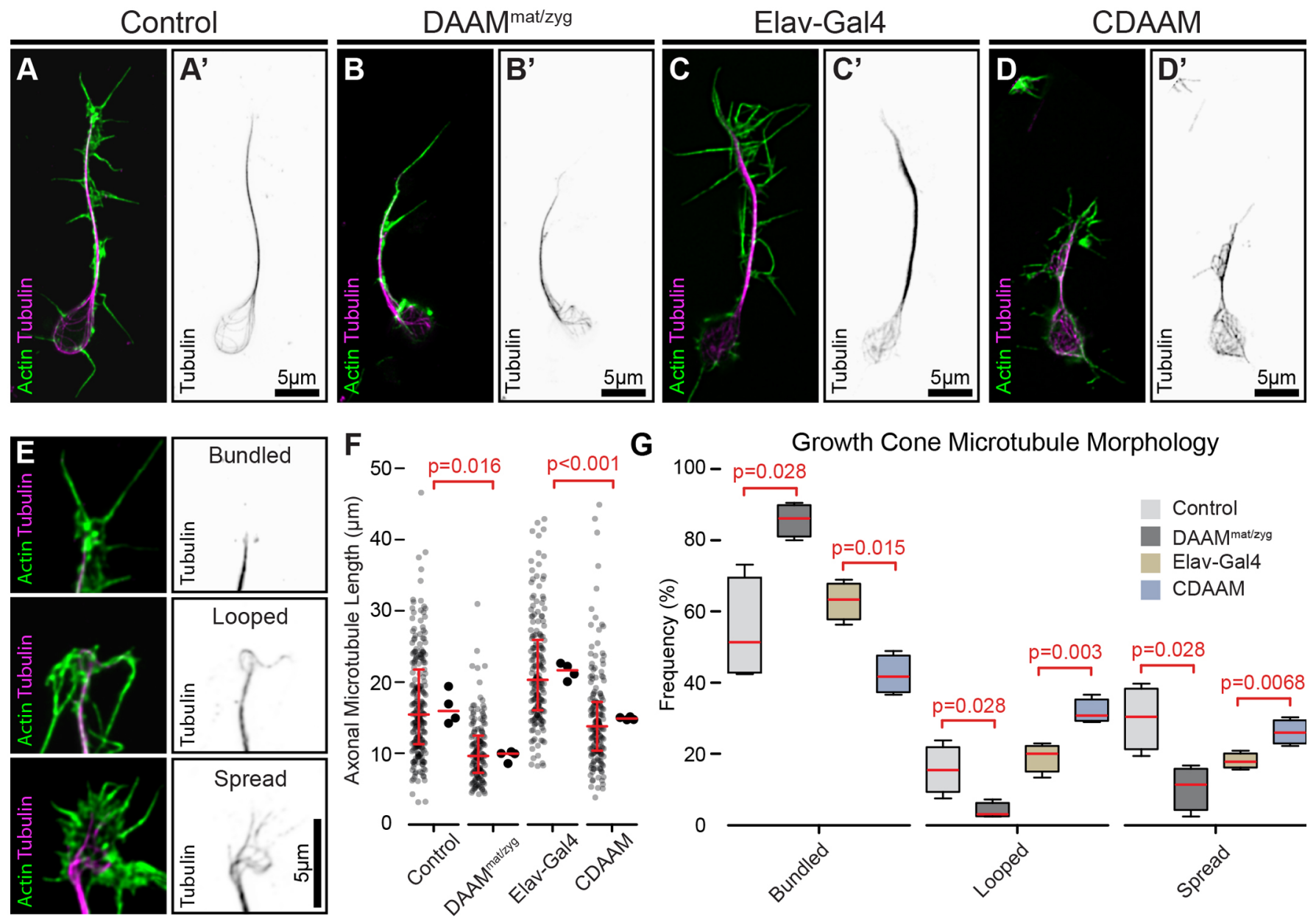

Fig. 2. DAAM affects axonal microtubule length and morphology. (A-D) Representative images of primary neurons $(6 \mathrm{HIV})$ derived from wild type ( white ${ }^{1118}$ ) $\left(\mathrm{A}, \mathrm{A}^{\prime}\right), D A A M^{\text {mat/zyg }}$ (see Materials and Methods) (B, $\left.\mathrm{B}^{\prime}\right)$, embryos expressing Elav-Gal4 (w;elav-Gal4/+) (C, $\left.\mathrm{C}^{\prime}\right)$ or embryos expressing CDAAM (w; elav-Gal4, UAS-CDAAM/+) (D, $\left.\mathrm{D}^{\prime}\right)$. (E) Examples of growth cone microtubules classified as being bundled, looped or spread. (F) Scatterplots show the length of the axonal microtubules of primary neurons. Gray dots represent values measured on individual cells (wild type: $15.42 \mu \mathrm{m}, n=235 ; D A A M{ }^{\text {mat/zyg: }} 9.61 \mu \mathrm{m}, n=152$; Elav-Gal4: $20.3 \mu \mathrm{m}, n=182$; CDAAM: $13.8 \mu \mathrm{m}, n=178$; median with interquartile range). Black dots represent the median of the independent experiments with their median. (G) Boxplots show the frequency of axonal microtubule morphology classes. Unpaired $t$-tests were used for statistical analysis.

quantified by using ImageJ's TrackMate plugin (Tinevez et al., 2017), following live acquisition of high-resolution images of neurons at 6-9 hours in vitro (HIV) expressing EB1::GFP, a marker of MT plus-ends (Morrison et al., 2002; Stepanova et al., 2003) (Fig. 3A,B and Fig. S3H). Time-lapse recordings were deconvolved and pre-filtered with a custom-made difference of Gaussian filter to enhance the signal of interest and to exclude false positive hits (Fig. S3A-B"; Movie 2). The analyses of EB1::GFP track velocity in wild-type neurons revealed that MTs tend to slow down upon entering the growth cones, showing a slight but consistent growth velocity difference between the growth cone $\left(6.54 \mu \mathrm{m} \times \mathrm{min}^{-1}\right)$ and the axonal shaft MTs $\left(8.12 \mu \mathrm{m} \times \mathrm{min}^{-1}\right)$ (Fig. 3C). For this reason, in the following studies, MT dynamics in growth cones and axonal shafts were analyzed separately. Because EB1 is known to promote MT growth by preventing MT catastrophes (Komarova et al., 2009), as a further control, we compared MT growth velocity in cultured neurons expressing either EB1::GFP or Jupiter::GFP (another widely used marker of microtubule dynamics) (Karpova et al., 2006). We measured the dynamic instability parameters of individual MTs in the growth cone and found no significant differences between EB1::GFP- or Jupiter::GFP-(6.47 $\pm 1.5, n=8)$ expressing neurons (Fig. S3C-F) and, hence, used EB1::GFP to monitor MT dynamics in all our subsequent studies.

First, we compared microtubule growth velocities in control and $D A A M^{\text {mat } / z y g}$ mutant neurons. We found that the median growth velocity measured in $D A A M^{\text {mat } / z y g}$ mutant neurons was $\sim 91 \%$ higher in the growth cones and $\sim 80 \%$ higher in the axon shaft compared to the controls ( $P<0.0001$ for both) (Fig. 3D; Movie 3). Parallel to that, lifetime measurements of growth cone MTs by utilizing the KaplanMeier estimator, revealed a reduction in MT lifetime of the mutant neurons $(P<0.0001)$ (Fig. 3E). The presence of DAAM seems to reduce MT growth rate and enhance lifetime by decreasing catastrophe frequency. Thus, these experiments showed that $D A A M$ affects MT dynamics in neurites, which is likely to be the cause of the altered MT organization phenotypes observed previously. In line with former observations by Bartolini et al. (2008) for mDia2, it appears that increased MT dynamics is paralleled with reduced lifetime, which would be consistent with a role in MT stabilization.

DAAM is involved in retrograde translocation of microtubules in growth cone filopodia

To better understand the mechanisms of how DAAM affects MT dynamics, we focused our attention on the individual pioneer 

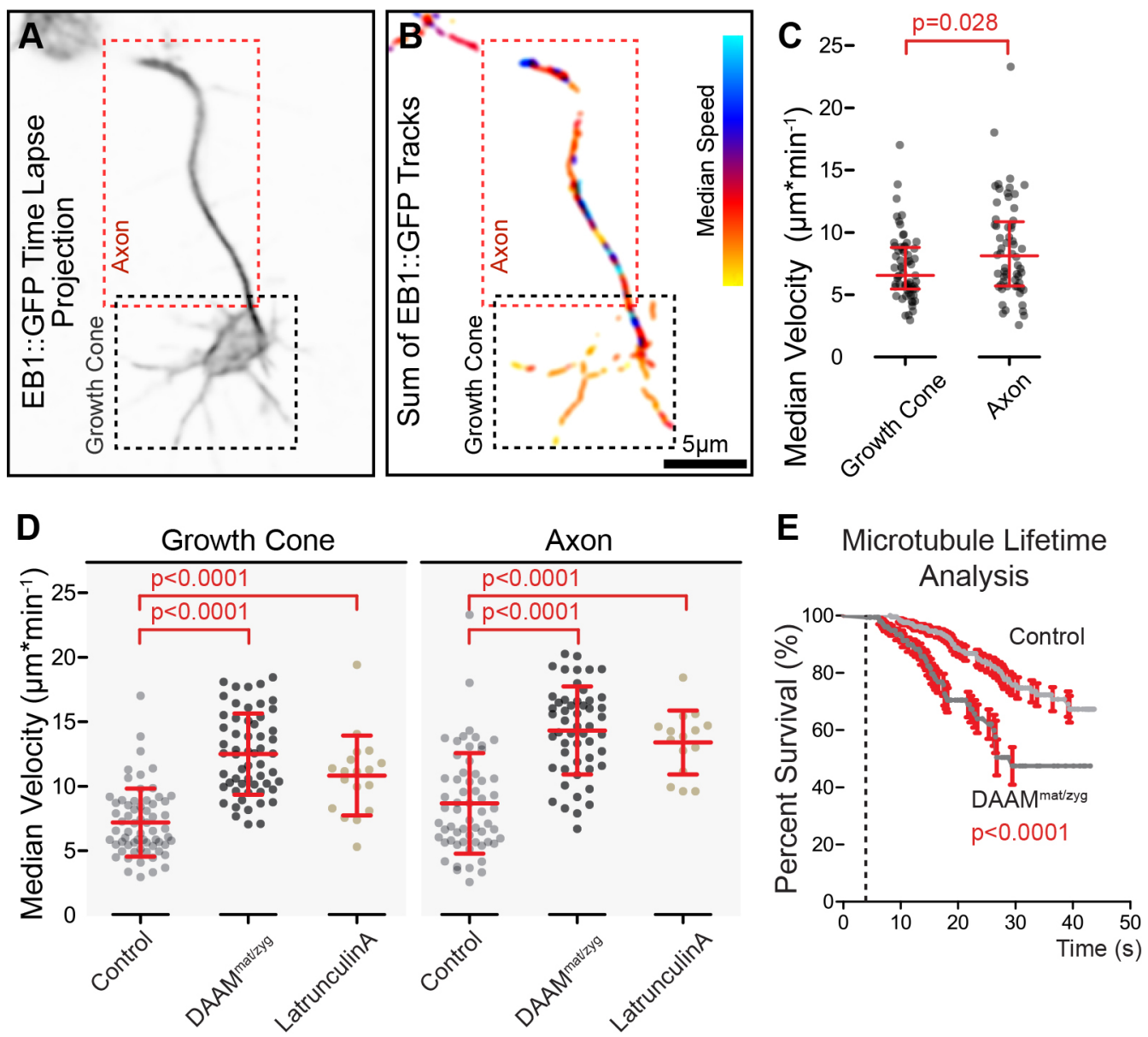

Fig. 3. The effect of DAAM on axonal microtubule dynamics. (A) Representative image of a primary neuron expressing EB1::GFP (6 HIV) (time-lapse projection). The axon and the growth cone are surrounded by dotted rectangles. (B) Time-lapse projection of recognized EB1::GFP tracks. The tracks are color-coded according to their median velocity (from yellow to cyan, $0 \mu \mathrm{m} \times \mathrm{min}^{-1}-30 \mu \mathrm{m}^{-1} \mathrm{~min}^{-1}$ ). (C) Scatterplots show significant velocity difference between the axonal and growth cone EB1::GFP comets (growth cone: $6.54 \mu \mathrm{m} \times \min ^{-1}, n=64 ;$ axon: $8.12 \mu \mathrm{m} \times \mathrm{min}^{-1}, n=60$; median with interquartile range). (D) Scatterplots show the median velocity of EB $1:: G F P$ tracks measured on primary neurons derived from EB1::GFP-expressing wild-type and $D A A M$ mat/zyg embryos. It is also compared to EB1::GFP-expressing wild-type neurons treated with latrunculin A (growth cone - wild type: $6.54 \mu m \times$ min ${ }^{-1}, n=64 ; D A A M{ }^{\text {mat/zyg. }}$

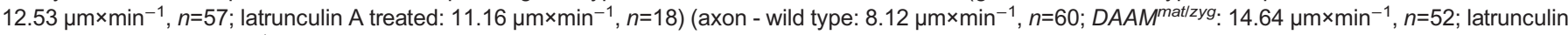
A treated: $13.85 \mu \mathrm{m} \times \mathrm{min}^{-1}, n=15$; median with interquartile range). Dots represent the median velocity of the EB1::GFP tracks measured on individual cells. Mann-Whitney test was used to compare the respective pairs. (E) Survival analysis was used to compare microtubule lifetime of wild-type and $D A A M$ mat/zyg neurons expressing EB1::GFP. Short tracks (below $3.6 \mathrm{~s}$ ) were discarded from the analysis and the threshold is marked with a dotted line. The Mantel-Cox test was used to compare the survival curves (white ${ }^{1118}, n=219 ; D A A M^{\text {mat/zyg }}, n=161$ ).

microtubules that extend into the growth cone periphery. Pioneer microtubules are of particular importance since their entry into filopodia and their subsequent stabilization is essential prior to growth cone turning (Buck and Zheng, 2002) and axon branching (Dent and Kalil, 2001). Expression of EB1::GFP is a suitable method to follow the plus-end of these MTs (Fig. 4A) as, in most cases, it is obvious when they enter filopodia and subsequently disappear at some point along their length, presumably because the MT has either stopped growing or got depolymerized. To follow pioneer MT growth, we recorded kymographs along the axis of filopodia, then we calculated the directionality distributions of these graphs to differentiate between anterograde displacement, pause and retrograde translocation (Fig. 4B-C $\mathrm{C}^{\prime}, \mathrm{E}^{-\mathrm{F}^{\prime}}$ and Fig. S3H-L). We observed that the EB1::GFP signal frequently paused and/or moved rearwards into the growth cone filopodia of wild-type cells (Fig. 4B, $\left.\mathrm{B}^{\prime}\right)$. Given that EB1 decorates only the polymerizing end of MTs, this suggests that these MTs undergo retrograde translocation which is a commonly observed phenomenon in the growth cone periphery (Schaefer et al., 2008). It is thought that MTs tend to couple to the retrograde actin flow through transient interactions and are transported back as they assemble (Marx et al., 2013) (consistently, in our primary neurons the retrograde MT translocation rate appears similar to that of retrograde actin flow, $\sim 5.3 \mu \mathrm{m} \times \mathrm{min}^{-1}$ ). Therefore, the retrograde actin flow poses a dynamic barrier to pioneer microtubules and removes them from the growth cone periphery (Schaefer et al., 2002, 2008). Remarkably, however, in $D A A M^{\text {mat } / z y g}$ mutant neurons we rarely observed paused or retrograde translocation of MTs into filopodia (Fig. $4 \mathrm{C}, \mathrm{C}^{\prime}$ ). The frequency of these events was significantly lower when compared to control cells $(P=0.02)$ (Fig. 4D-F'). Therefore, we conclude that, at least in the pioneer MTs, the lack of DAAM reduces the retrograde translocation rate of MTs that is consistent with the increased MT growth velocity.

\section{Direct versus indirect effects on microtubule dynamics upon loss of DAAM}

Since formins have originally been described as regulators of the actin cytoskeleton, the question arises whether microtubule 

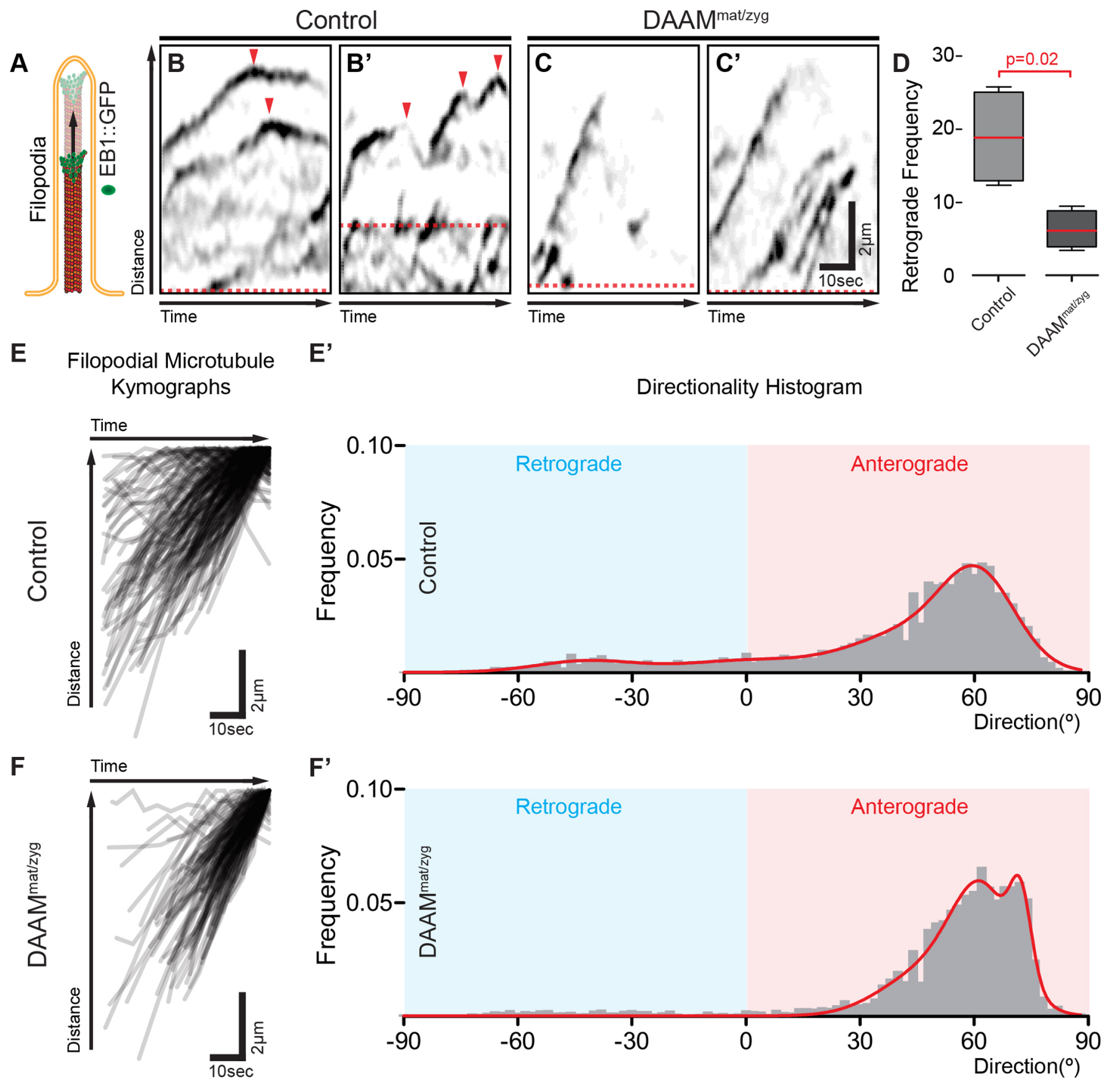

$F^{\prime}$
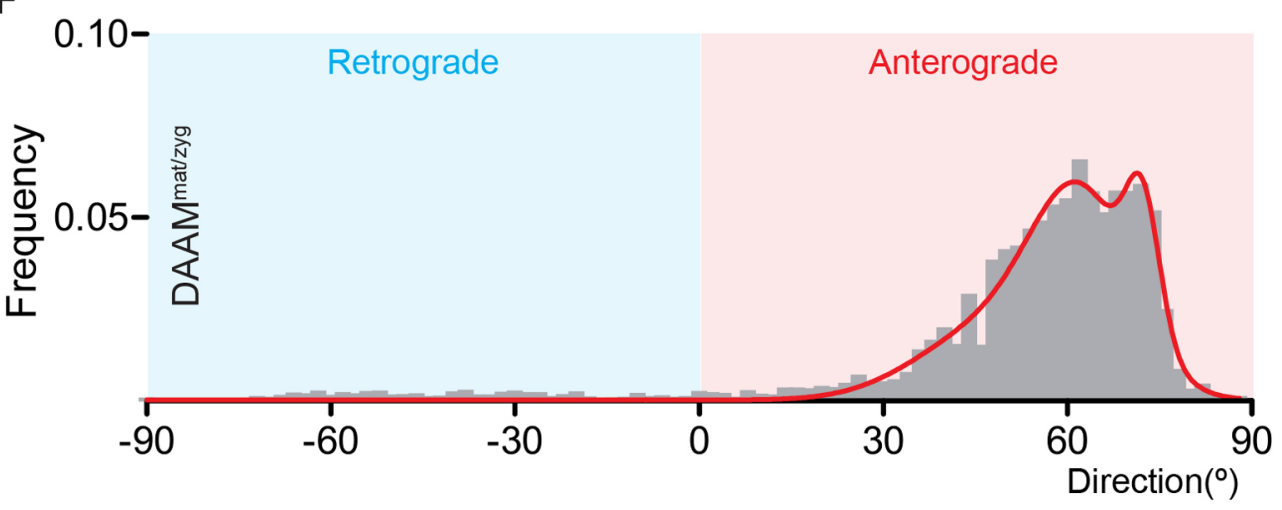

Fig. 4. The effect of DAAM on retrograde MT translocation. (A) The cartoon illustrates a growth cone filopodia invaded by a microtubule where the growing plus-end is labeled with EB1::GFP. (B-C') Representative kymographs demonstrate microtubule plus-end displacement along growth cone filopodia in wild-type $\left(B, B^{\prime}\right)$ and $D A A M^{\text {mat/zyg }}\left(C, C^{\prime}\right)$ neurons expressing EB1::GFP. Red arrowheads mark the point where microtubules stall or undergo retrograde translocation. Dotted lines indicate where pioneer microtubules enter the growth cone filopodia. (D) Boxplot shows the frequency of microtubule retrograde translocation in wild-type and $D A A M^{\text {mat/zyg }}$ neurons (white ${ }^{1118}: 18.9 \pm 6.4, n=219 ; D A A M^{\text {mat/zyg: }}$. $3 \pm 2.5, n=161 ;$ mean \pm s.d., from four independent experiments). (E-F') Summary of extracted kymographs from wild-type (E) and DAAM ${ }^{\text {mat/zyg }}(\mathrm{F})$ neurons expressing EB1::GFP. (E',F') Histograms show the summarized directionality distribution, measured on the extracted kymographs.

cytoskeleton-related phenotypes are direct effects of DAAM absence or the consequence of an impaired actin cytoskeleton. To elucidate the role of the actin cytoskeleton on MT dynamics, we disturbed the actin cytoskeleton by applying latrunculin A, a drug that sequesters G-actin and promotes depolymerization (Coué et al., 1987; Yarmola et al., 2000). Primary neurons expressing Actin5C:: GFP treated with $200 \mathrm{nM}$ latrunculin A showed signs of actin cytoskeleton breakdown within the growth cone as early as $30-60 \mathrm{~s}$ after treatment. This depolymerization plateaus after $\sim 5$ min when the stereotypic searching movements of filopodia completely disappear (Fig. S4A-D; Movie 4). After establishing the F-actin breakdown protocol, we applied the same conditions to primary neurons expressing EB1::GFP and measured MT growth dynamics during 5-20 min after treatment. We found that median growth velocity is increased both in the axon shaft and the growth cone region (Fig. 3D; Movie 5). Although this increase was similar to the 
one observed in $D A A M^{\text {mat/zyg }}$ neurons, we noticed the formation of long, slender microtubule-containing protrusions, which was not observed in DAAM $M^{\text {mat/zyg }}$ neurons (Fig. S4E-I; Movie 5). These results indicate that the F-actin network forms a barrier to MT growth in axons - like the one already reported in growth cones (Burnette et al., 2007; Forscher and Smith, 1988; Zhou and Cohan, 2004) - but seems to apply to the shaft as well. Although the latter might imply the existence of an unexplored actin/MT crosstalk in axonal shafts, we subsequently focused our studies on the peripheral growth cone.

We reasoned that changes in MT dynamics upon loss of DAAM might be the result of a slower actin retrograde flow or a change in the coupling of microtubules to the retrograde flow, or a combination of these two effects. To begin to test these alternatives, we compared the retrograde actin flow in filopodia of wild-type and $D A A M$ mutant neurons. First, we followed growth-cone motility by measuring filopodial dynamics in neurons expressing Actin5C::GFP (Movie 6). Live recordings (7-9 HIV) revealed that the Actin5C:: GFP signal was slightly fainter in $D A A M^{\text {mat } z y g}$ neurons as compared to wild-type neurons and, in accordance with previous findings (Matusek et al., 2008), filopodia number and length were reduced (Fig. 5B,C). To quantify the dynamic properties of growth cone filopodia, we recorded kymographs along the axis of the filopodia and calculated their extension and retraction velocity based on their tip displacement as a function of time. The mean extension velocity was elevated by $28 \%$ (not significant), whereas the mean retraction rate decreased by $33 \%(P=0.0096)$ in the $D A A M^{\text {mat } / z y g}$ mutant neurons as compared to wild type (Fig. 5E,F). Moreover, by analyzing the fluorescent speckle pattern (Waterman-Storer and Salmon, 1998) (Fig. 5D,D') of the Actin5C::GFP signal, we determined that the mean velocities of the retrograde actin flow remained constant when the filopodia extended or retracted (Bornschlogl et al., 2013). The kymographs also revealed that the rate of the retrograde flow highly correlates with the retraction rate of the filopodia, both in wild-type and $D A A M^{\text {mat } / z y g}$ neurons. Consistent with the reduced filopodia retraction rate, retrograde flow is decreased by $37.5 \%$ (not significant) in $D A A M^{\text {mat } / z y g}$ neurons (Fig. 5G). Since extension and retraction rates of the growth cone filopodia depend on the retrograde flow and the growth rate of the filopodial F-actin bundle, i.e. the polymerization rate of newly incorporated actin at the filopodial tip, we could calculate the plusend dynamics of the actin filaments during filopodia extension and retraction (Fig. 5A,H). We found that, during filopodia extension, the plus-end growth of actin filaments exceeds the retrograde flow, and that this rate is $20 \%$ faster (not significant) in $D A A M^{\text {mat } / z y g}$ neurons as compared to wild type (Fig. 5I). In most filopodia the plus-end of the actin filament appears to be stalled during retraction both in wild-type and $D A A M^{\text {mat/zyg }}$ neurons (Fig. 5J); therefore, the retraction completely depends on the retrograde flow.

In summary, we revealed that, although the actin-depolymerizing drug latrunculin A had effects on MT growth dynamics that are similar to the lack of $D A A M$, it also induced the formation of long, filopodia-like protrusions that have never been observed in DAAM mutant neurons and, henceforth, these effects are clearly not identical. Moreover, we found that the absence of DAAM has a very weak effect on the retrograde actin flow, whereas the retrograde MT translocation frequency exhibits a considerable reduction in the mutant neurons. Therefore, these data suggest that the effect of DAAM on MT dynamics is unlikely to be an indirect (or largely indirect) effect due to altered actin dynamics (i.e. reduced retrograde actin flow); instead, they might indicate a failure in the correct coordination of actin and MT dynamics.

\section{DAAM stabilizes microtubules in vitro and in vivo}

Consistent with findings for some other members of the formin protein family (Bartolini et al., 2008; Chesarone et al., 2010; Gaillard et al., 2011), DAAM appears to promote MT stabilization in primary neurons as suggested by our MT dynamics studies. To test this hypothesis more directly, we examined whether DAAM protects against MT destabilization under two different conditions. First, an MT co-sedimentation assay was used to measure the effect of cold treatment on preassembled MTs in the presence or absence of purified GST::FH1FH2 protein. According to our expectation, MTs were protected against cold-induced depolymerization in the presence of FH1FH2, indicated by elevated tubulin levels in the pellet (Fig. 6D, E). Second, as a parallel approach, we asked how loss of DAAM affects MT stability. To this end, we induced MT depolymerization by treating $D A A M^{\text {mat/zyg }}$ and control (derived from white ${ }^{1118}$ embryos) primary neuronal cultures with nocodazole. Control cells treated with nocodazole exhibited only a few areas along the axon which were devoid of microtubules (Fig. 6A-A' and C). In contrast, nocodazoletreated $D A A M^{\text {mat } / z y g}$ primary neurons had a twofold increase in the number of breaks along axonal microtubules (Fig. 6B-B' and C). In conclusion, DAAM behaves as a potent MT-stabilizing factor in two diverse assays, and its N-terminally truncated FH1FH2 form already seems sufficient to stabilize MTs, at least under in vitro conditions.

\section{DAAM mediates the alignment of microtubules and actin filaments}

To test the possibility of DAAM-mediated coupling of MTs and F-actin, we performed in vitro low-speed sedimentation experiments adapted from Elie et al., 2015 (Fig. 7A-C). Phalloidin-stabilized F-actin and/or taxol-stabilized MTs were centrifuged onto a sucrose cushion in the absence or presence of DAAM constructs. This strategy ensures that individual polymers (F-actin or MT) and F-actin bundles assembled by DAAM remain in the supernatant, as only larger polymer complexes deposit as sediment and appear in the pellet (Elie et al., 2015). Accordingly, in the presence of GST:: CDAAM MTs were detected in the pellet, whereas in the presence of both F-actin and MTs a fraction of F-actin co-sedimented with microtubules in the GST::CDAAM-containing samples (Fig. 7C). Intrinsic to our experimental conditions, F-actin can only appear in the pellet when it is physically linked to MTs; therefore, these observations imply a simultaneous interaction of CDAAM and the two polymer systems. In contrast to CDAAM, actin or MT polymers did not sediment in the presence of GST::FH1-FH2 or the C-terminal DAD-CT, DAD or DAD-CT ${ }^{\mathrm{R}-\mathrm{A}}$ constructs (Fig. 7C and Fig. S4J), suggesting that the $\mathrm{FH} 2$ and the $\mathrm{CT}$ regions are both required for F-actin and MT crosslinking, yet neither is sufficient alone.

To further test the F-actin/MT coordinating ability of DAAM, total internal reflection fluorescence microscopy (TIRFM) was carried out with fluorescently labeled F-actin (labeled with AlexaFluor-568NHS) and MTs (labeled with HiLyte Fluor ${ }^{\mathrm{TM}}$ 488) (Fig. 7D-F"'). The F-actin area colocalizing with MTs, as well as the MT area colocalizing with F-actin was quantified (Elie et al., 2015) (Fig. 7G,H). In control samples lacking DAAM, $\sim 13 \%$ of F-actin and MTs appeared to be co-aligned $(n=19)$ (Fig. 7D-D"'), and the presence of GST::FH1-FH2 did not change this percentage $(n=18)$ (Fig. 7F-F'"'). By contrast, GST::CDAAM increased the fraction of co-aligned polymers to $\sim 40 \%(n=22)$ (Fig. 7E-E"' $)$, corroborating that DAAM promotes actin and MT co-alignment. In agreement with this, our actin/microtubule/DAAM colocalization studies revealed that $\sim 22 \%$ (PPI: $0.224 \pm 0.04$ ) of DAAM is associated with both the actin and MT cytoskeletons (Fig. 8A-D), 

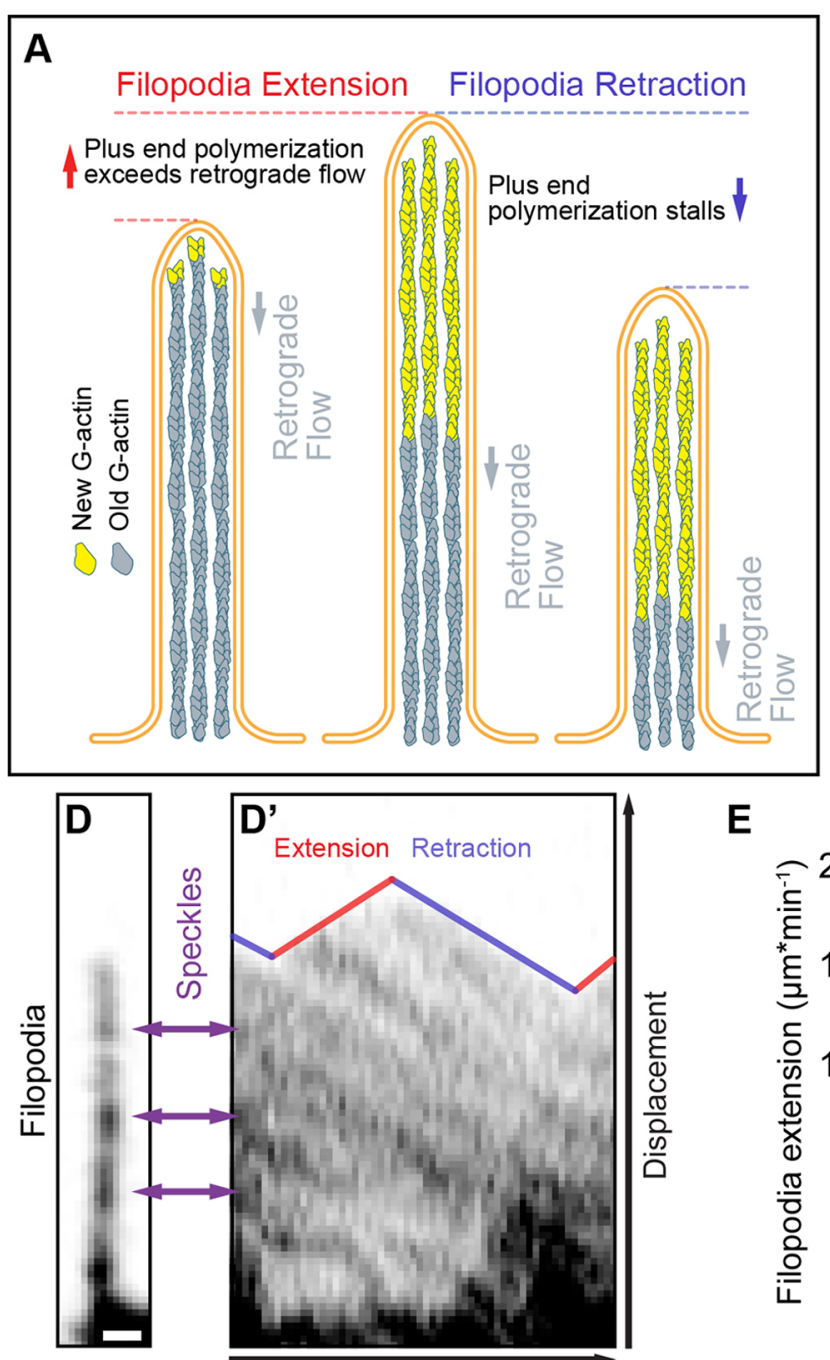

Time

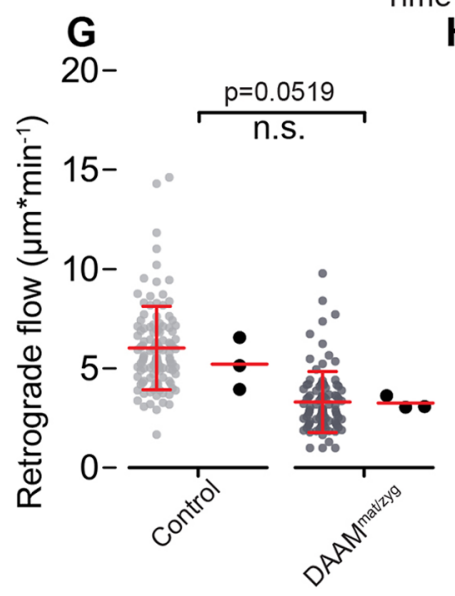

E

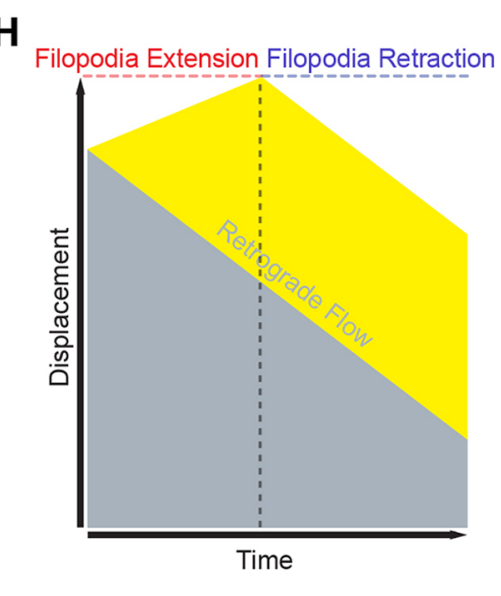

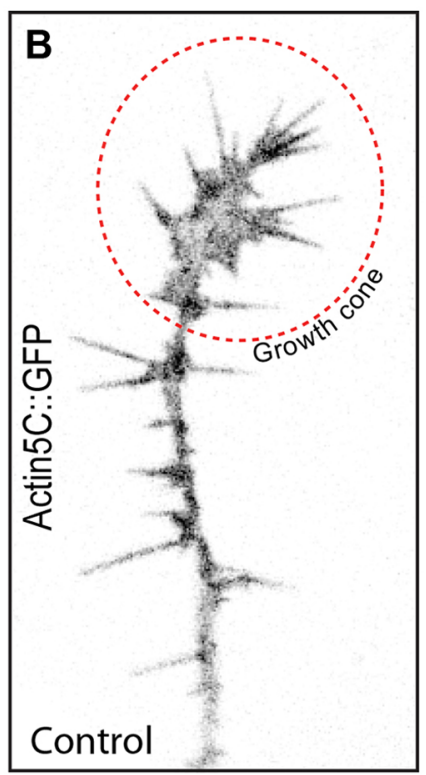

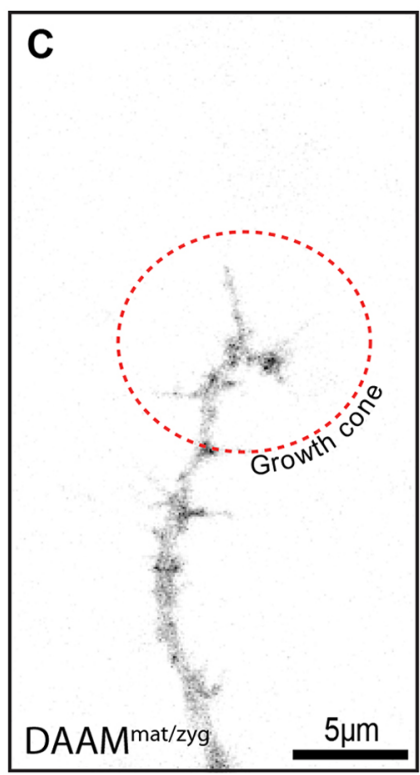

$\mathbf{F}$
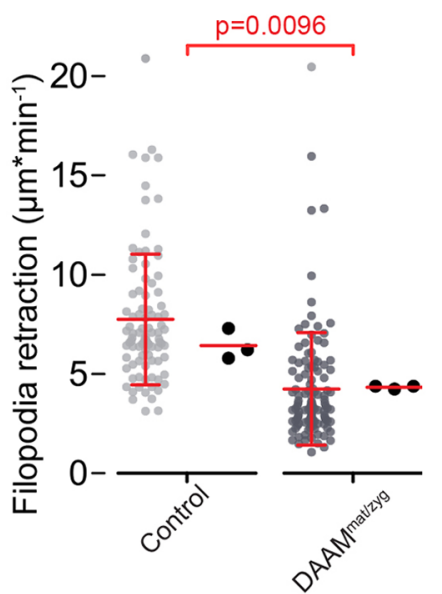

I

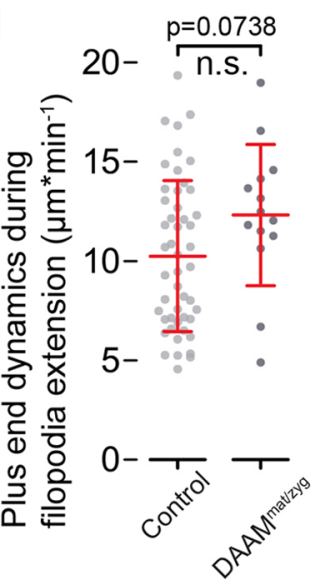

J

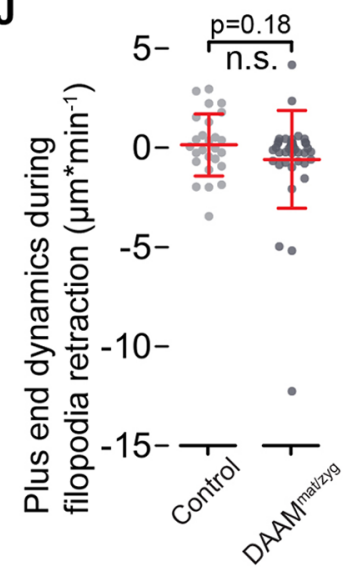

Fig. 5. Effect of DAAM on axonal filopodia and actin dynamics. (A) A drawing to illustrate filopodia dynamics (extension and retraction) and the

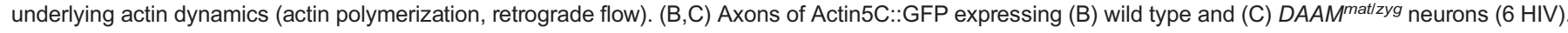
Growth cones are indicated by a dotted red circle. (D) Filopodia with unevenly incorporated Actin5C::GFP; scale bar: $200 \mathrm{~nm}$. (D') Kymograph revealing the actin retrograde flow meanwhile the filopodia extends and retracts. (E-G) Scatterplots show filopodia extension (wild type: $5.28 \mu \mathrm{m}^{2} \mathrm{~min}^{-1}, n=134 ; D A A M^{\mathrm{mat}}$ zyg: $6.75 \mu \mathrm{m} \times \mathrm{min}^{-1}, n=64$ ), retraction (wild type: $6.82 \mu \mathrm{m} \times \mathrm{min}^{-1}, n=91 ; D A A M^{\text {mattzyg: }} 3.38 \mu \mathrm{m} \times \mathrm{min}^{-1}, n=117$ ) and actin retrograde flow (wild type: $5.64 \mu \mathrm{m} \times \mathrm{min}^{-1}$,

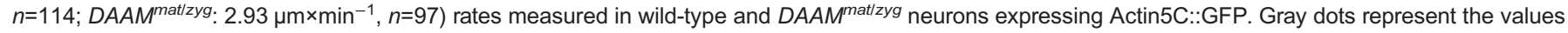
of individual events. Black dots represent the median of independent experiments. Mann-Whitney test was applied for statistical analysis. (H) Scheme of a hypothetical kymograph recorded along filopodia depicted on panel A. (I,J) Scatterplots show actin plus-end dynamics during filopodia extension (wild type: $5.28 \mu \mathrm{m} \times \mathrm{min}^{-1}, n=134 ; D A A M^{\text {mattzyg: }} 6.75 \mu \mathrm{m} \times \mathrm{min}^{-1}, n=64$ ) and retraction rates (wild type: $6.82 \mu \mathrm{m}^{2} \times \mathrm{min}^{-1}, n=91 ; D A A M^{\text {mattzyg: }} 3.38 \mu \mathrm{m}^{2} \times \mathrm{min}^{-1}, n=117$ ) measured in wild-type and $D A A M^{\text {mat/zyg }}$ neurons expressing Actin5C::GFP. Gray dots represent the values of individual events calculated based on the actin retrograde flow and filopodia extension and retraction rates. 

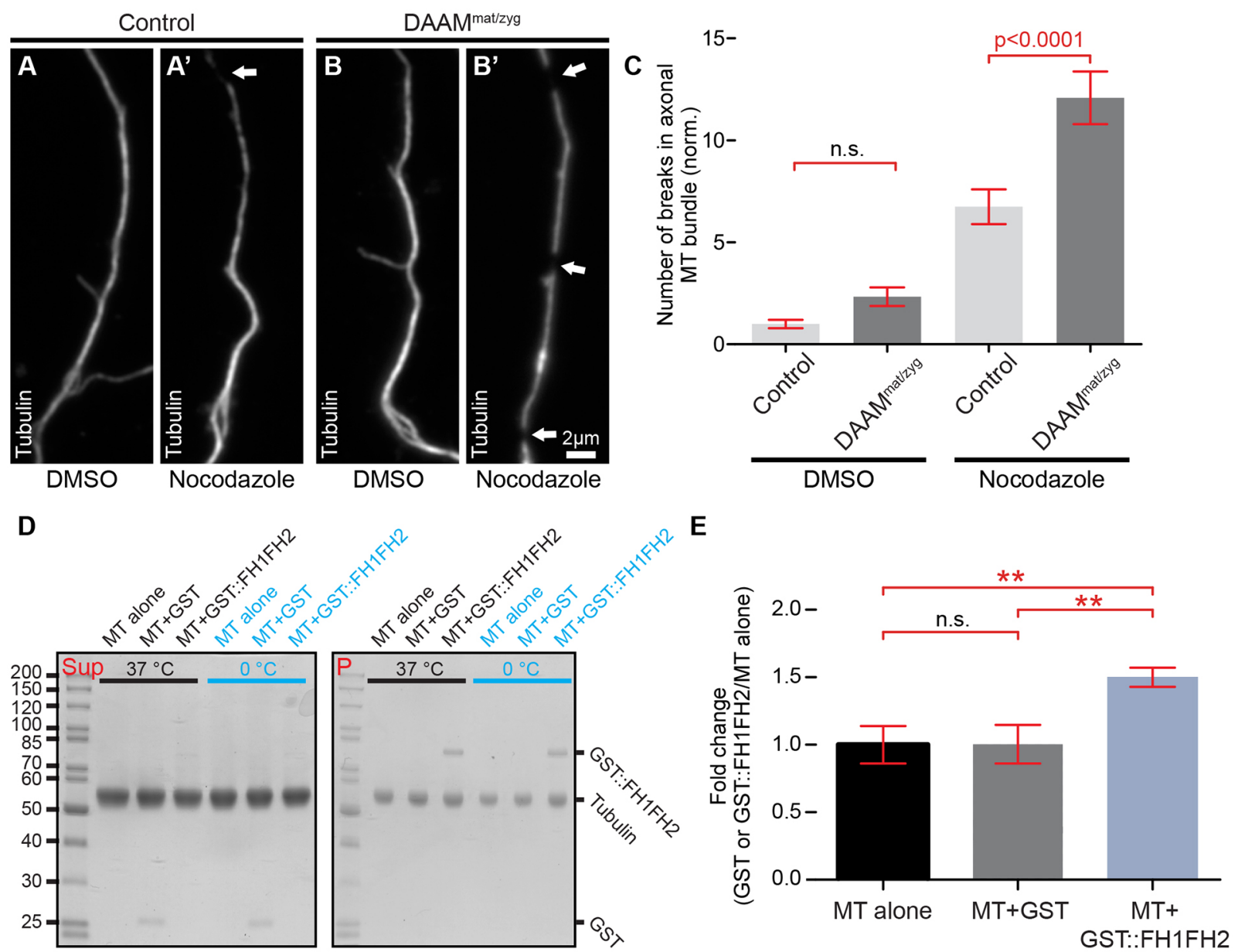

Fig. 6. DAAM stabilizes MTs in vivo and in vitro. (A-B') Images show axonal MT bundles of DMSO-treated (A,B) or nocodazole-treated ( $\left.\mathrm{A}^{\prime}, \mathrm{B}^{\prime}\right)$ control (white ${ }^{1118}$ ) and DAAM ${ }^{\text {mat/zyg }}$ primary neurons (12 HIV). Arrows point to gaps along axonal MT bundles. (C) Quantification of the number of breaks along the MT bundles of control (DMSO treated: $1 \pm 0.2$, mean \pm s.e.m., $n=213$; nocodazole treated: $6.7 \pm 0.8$, mean \pm s.e.m., $n=206$ ) and $D A A M{ }^{\text {mat/zyg }}$ primary (DMSO treated: 2.3 \pm 0.4 , mean \pm s.e.m., $n=157$; nocodazole treated: $12 \pm 1.3$, mean \pm s.e.m., $n=137$ ) neurons, measured in three independent experiments. (D,E) Densitometric analysis of SDS-PAGE (D) show that the amount of tubulin in the pellet is higher in samples with GST::FH1FH2 (E) after cold-induced depolymerization as compared to controls (mean \pm s.d., $n=3$, ${ }^{* *} P<0.01$; n.s., not significant).

which means that the majority of MT-associated DAAM ( 37\%) also colocalizes to the actin cytoskeleton. The capacity of DAAM to simultaneously bind the two cytoskeletal systems in vitro together with its axonal localization along the overlapping regions, strongly suggest that DAAM is involved in the coordination of the actin and MT cytoskeleton.

\section{DISCUSSION}

Proper coordination of the actin and microtubule cytoskeleton is thought to be key to growth cone advance, yet the molecular mechanisms of actin-MT crosstalk during axonal growth remained largely elusive. Here, we report our findings, suggesting that the formin family protein DAAM is an important factor of actinmicrotubule coordination in neuronal growth cones. Formins are extensively characterized for their ability to interact with G-actin and promote their assembly into F-actin (Higashida et al., 2004; Kovar and Pollard, 2004; Watanabe and Higashida, 2004). These studies established that the FH2 domain binds actin strongly, whereas some formins contain an additional, albeit much weaker actin-binding motif in their DAD-CT region (Gould et al., 2011; Vizcarra et al., 2014). In accordance with recent findings for other formins, we found that, besides actin binding (Barko et al., 2010), the FH2 domain of DAAM is also able to interact with MTs. Moreover, we showed that the positively charged $\mathrm{CT}$ region but not the DAD domain is also capable of MT binding. Considering the dimeric nature of the formin proteins, the full-length DAAM protein contains multiple actin- and MT-binding sites. However, how these interacting elements act in a concerted fashion is still elusive. The CDAAM fragment that contains all the mapped cytoskeletoninteracting domains of DAAM exhibits an F-actin-bundling activity in vitro and is also able to crosslink actin filaments with MTs. In the concentration range where purified CDAAM is able to co-align Factin and MTs, neither FH1-FH2 nor DAD-CT possesses these activities. This suggests a synergic action of these regions, for which the polymer-binding ability of the $\mathrm{FH} 2$ domain seems to be substantial. On the basis of these in vitro data the question arises which of these activities are relevant in vivo and, particularly, in growth cones. With regard to actin bundling, although not distributed uniformly, DAAM is detected along the filopodial actin bundles, which would be consistent with it having a role in filament bundling. Whereas the majority of DAAM associates either with actin filaments or with MTs, it is remarkable that a high amount of DAAM is colocalized with actin and MTs in growth cones, possibly indicating a role in crosslinking or co-alignment of actin and MTs. To further support this, the effect of DAAM on MT dynamics and organization can also be explained by the assumption that, in the 
A

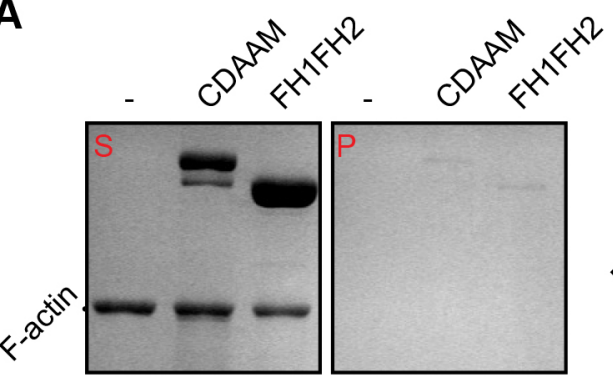

B

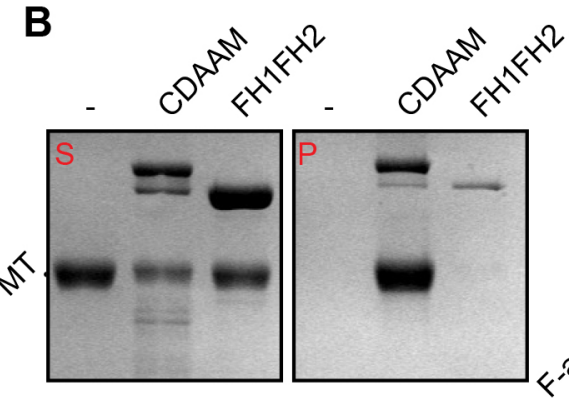

C
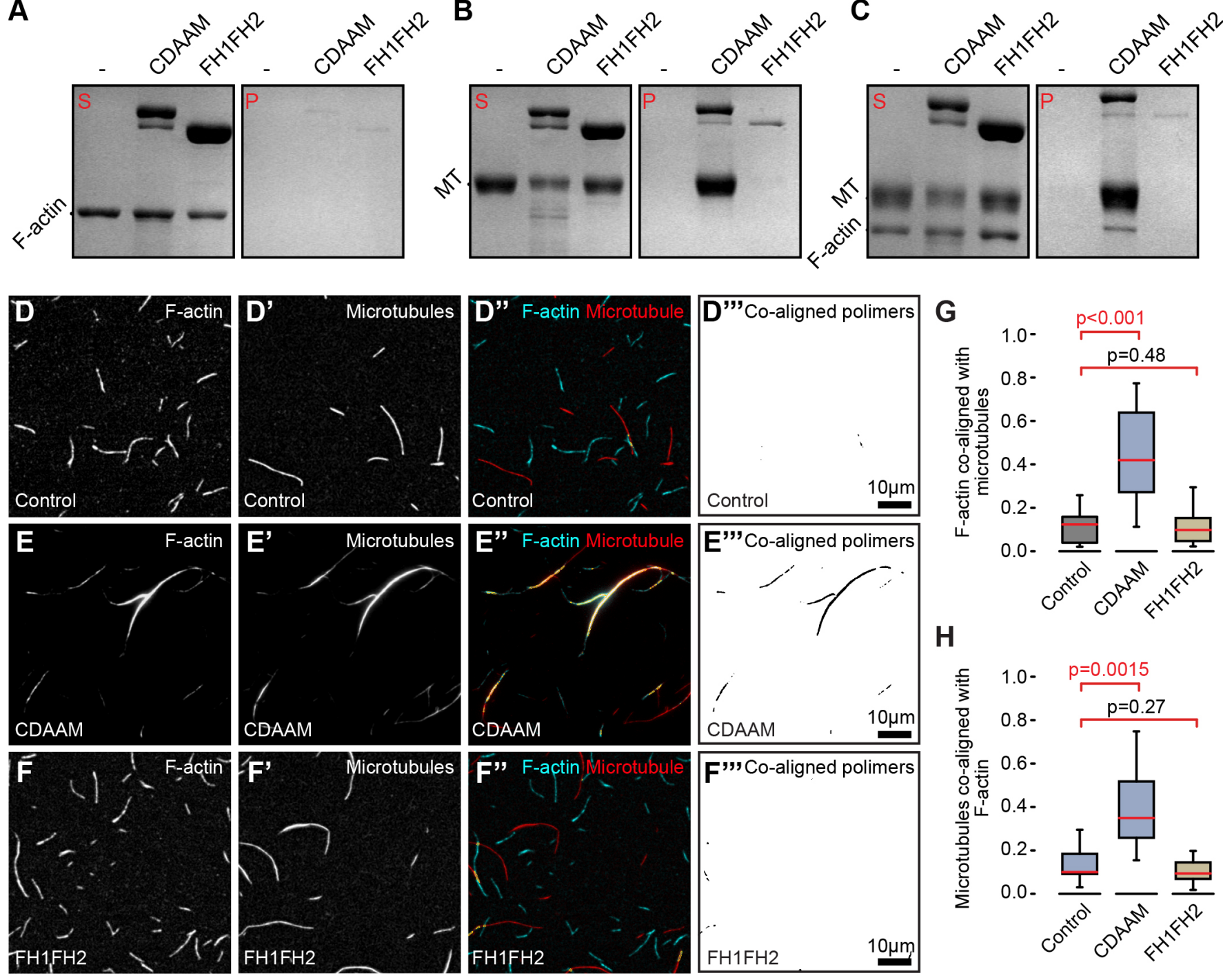

Fig. 7. F-actin and microtubule co-alignment mediated by DAAM. (A-C) Representative Coomassie Blue-stained SDS-PAGE from low-speed centrifugation experiments showing the amount of MTs and F-actin in the supernatants $(S)$ and in the pellets $(P)$ in the absence or presence of either GST::CDAAM or FH1-FH2, as indicated. (D-F) Representative fluorescence micrographs of F-actin (cyan) and MTs (red) in the absence or presence of GST::CDAAM and GST::FH1FH2, as indicated. Yellow regions on the merged images highlight the overlapping F-actin and MT regions. Binary images show the overlapping polymer area. $(\mathrm{G}, \mathrm{H})$ Ratio of the co-aligned F-actin and MT areas in the absence or presence of GST::CDAAM or GST::FH1FH2 (average of three to four independent experiments is shown).

absence of $D A A M$, the two cytoskeletal systems are disconnected from each other.

Organization of the actin and MT cytoskeleton is intimately linked in most cellular processes examined to date. Therefore, perturbation of one of the two systems is likely to indirectly affect the other one as well. It follows that actin regulators, such as formins, might be able to affect cellular MT organization by indirect mechanisms in addition to or instead of direct effects. This has, so far, been an often ignored, yet critical question when studying the role of formins in MT regulation. We attempted to address this issue by comparing the effect of the actin-depolymerizing drug latrunculin A with the lack of DAAM in primary neurons. Upon latrunculin A treatment, we found accelerated growth of MTs in the growth cone, which was very similar to what is observed in the absence of DAAM. However, we also noticed that latrunculin A treatment induced the formation of long filopodia-like protrusions that had not been detected previously in DAAM mutant cells. Thus, it appears that partial depolymerization of the neuronal actin cytoskeleton has an effect on MT dynamics that is comparable, yet not identical, to the loss of formin function, suggesting that an indirect effect cannot be excluded. Nevertheless, a number of observations argue against the idea that the effect of DAAM on MT organization within growth cones and dynamics would be exclusively or mainly indirect. First, DAAM is often colocalized with MTs and it clearly has the ability to bind to MTs. Second, DAAM is able to associate with MTs even when its actin binding is compromised. Third, the loss of DAAM strongly reduces the retrograde MT translocation frequency but only weakly affects the retrograde actin flow. These results, together with that of the actin/ MT crosslinking activity detected in vitro and the DAAM/actin/MT triple colocalization observed in growth cones, strongly suggest that the main function of DAAM is related to the coordination of actin and MT dynamics.

One intriguing characteristic of the interaction between DAAM and microtubules is the ability of DAAM to bind MTs directly as well as to associate with MTs through +TIPs. The main MT-related 

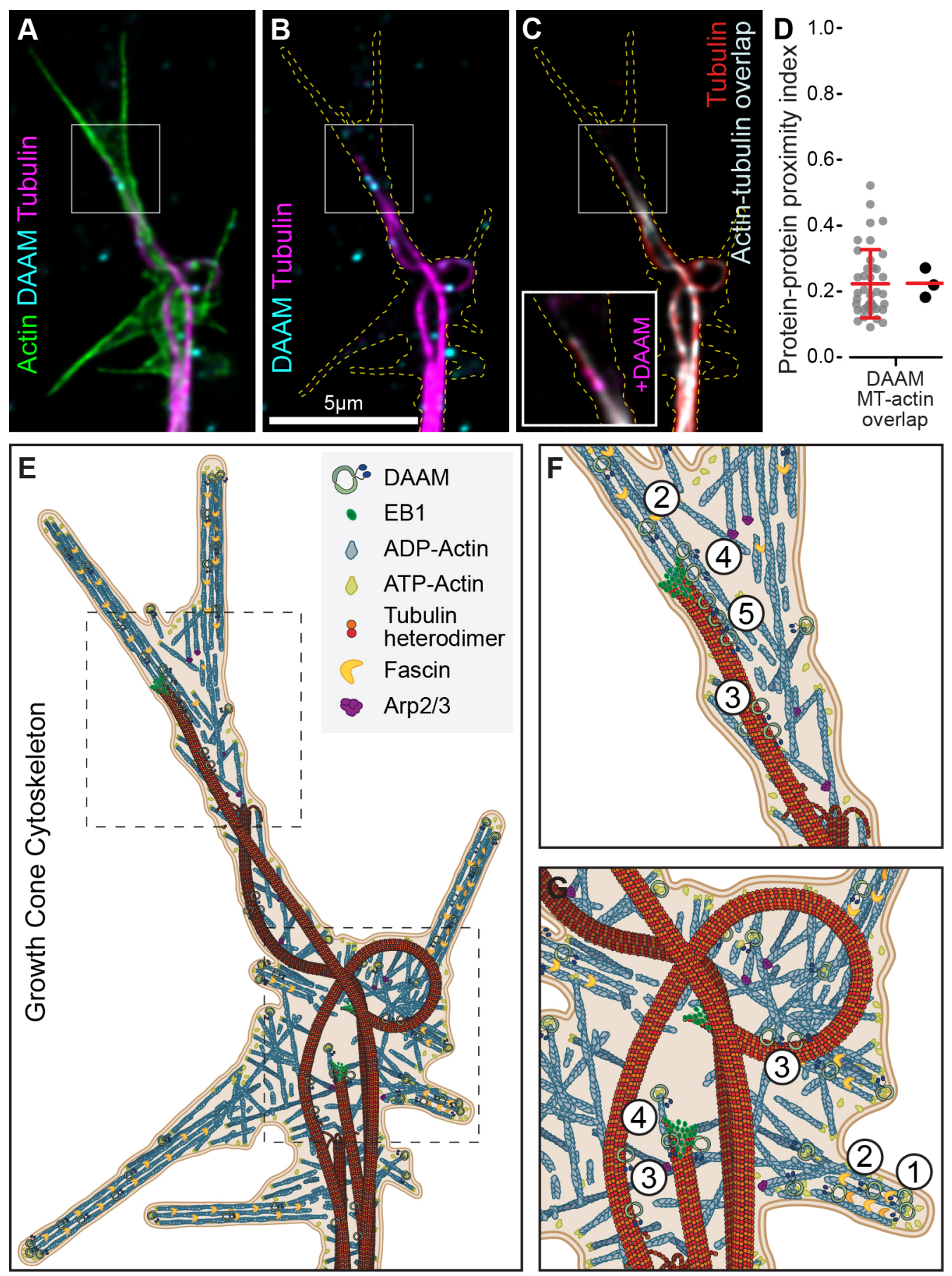

Fig. 8. DAAM colocalizes with overlapping F-actin and microtubules. (A-D) Representative growth cone showing DAAM puncta on overlapping F-actin and MTs (white rectangle). (B) Outline of the growth cone indicated by yellow dotted line. (C) Microtubules (red) and microtubules overlapping with actin filaments (gray). Inlet shows DAAM puncta (magenta) localized on overlapping F-actin and microtubules. (D) Scatterplots show the protein-protein proximity index measured between DAAM and the overlapping F-actin and microtubules. Gray dots represent values measured on individual cells $(0.22 \pm 0.1$, mean \pm s.d., $n=40)$. Black dots represent the median of the independent experiments with their median. (E) Schematic model of the growth cone cytoskeleton. (F,G) Localization and proposed functions of DAAM in growth cones on the basis of previous studies and this current study: (1) actin barbed-end binding, actin assembly,

(2) F-actin bundling, (3) MT side binding (stabilization), (4) EB1 binding, (5) F-actin and MT coalignment. Our results suggests that the key function of DAAM is to facilitate/boost the formation of new actin filaments at the MT plus-ends through interactions with EB1.

effects of DAAM, such as MT stabilization and actin-MT crosslinking can, potentially, be explained by direct MT binding, which would, consequently, impact on MT dynamics. In addition, DAAM, bound to MTs, might interact with other microtubuleassociated proteins, which would offer further regulatory possibilities. However, +TIP binding is equally exciting, particularly in light of recently discussed models of growth cone advance (Bearce et al., 2015; Cammarata et al., 2016), and because of data that reveal how MT plus-ends trigger formin-dependent rapid actin assembly in vitro and in motile cells (Henty-Ridilla et al., 2016; Jaiswal et al., 2013b; Okada et al., 2010). While in the classic models of growth cone guidance actin dynamics is credited as the 
main driving force of motility and as the primary target of guidance signaling, subsequent studies suggest that the peripheral pioneer MTs also act as guidance sensors, leading to the hypothesis that the closely coupled regulation of actin and MT dynamics is at the heart of guidance signaling (Bearce et al., 2015; Cammarata et al., 2016; Coles and Bradke, 2015). Meanwhile, +TIPs - many of which are able to interact with both F-actin and MTs - were shown to act as signal transducers during growth cone guidance, and they emerged as key regulators of actin/MT coordination downstream of axon guidance signaling. In parallel to this, it was established that some +TIPs collaborate with the formin mDia1, which enables MT plus-ends to govern localized actin assembly in vitro and in fibroblast cells (Henty-Ridilla et al., 2016; Jaiswal et al., 2013b; Okada et al., 2010), and that the interaction of CLIP170 with an unknown formin is important for dendritic arbor formation in primary neurons (Henty-Ridilla et al., 2016). Given that DAAM is present at MT plus-ends and it associates with EB1 in Drosophila $\mathrm{S} 2$ cells, we propose that DAAM is a strong candidate to promote actin assembly in concert with +TIPs. Thus, our data reinforce the importance of a formin/+TIP module as a general mechanism to link actin and MT dynamics and, most importantly, we provide the first in vivo evidence of such a mechanism in axonal growth cones.

In addition to the potential to promote actin polymerization at MT plus-ends, we found that DAAM retains an MT-stabilizing activity both in vitro and in primary neurons, and the $\mathrm{FH} 2$ domain seems sufficient for MT stabilization. This is similar for several other formins and, therefore, it might be a shared feature of this protein family. Although the physiological importance of formin-mediated MT stabilization in the context of neuronal growth remains largely unknown, DAAM clearly appears to possess multiple capabilities to influence the cytoskeleton due to its actin nucleation and elongation, MT stabilization, MT plus-end-binding and actin-MT crosslinking activities. Strikingly, perhaps with the exception of actin assembly, many other +TIPs exhibit related biochemical properties. On the basis of this we propose that the unique or key function of DAAM in orchestrating the growth cone cytoskeleton is to facilitate the formation of new actin filaments at the plus-ends of pioneer MTs (Fig. 8E-G). We think that this step is controlled by the navigation cues that also coordinate a multitude of cytoskeletal responses, including other transient actin-MT interactions, through other actinand/or MT-binding proteins, such as +TIPs, to guide axons in a complex cellular environment. Whether DAAM is an essential factor in all neurons or whether formins are - in this context redundant, awaits future elucidations.

\section{MATERIALS AND METHODS}

\section{Fly stocks and genetics}

For mutant analysis $D A A M^{E x I}$ or $D A A M^{E x I}$;elav-Gal4,UAS-EB $1:: G F P$ and $D A A M^{E x 68}, U b i:: G F P / Y^{D p(1 ; Y) S z 280}$ Drosophila lines were crossed as described previously (Matusek et al., 2008). In addition, we used the following stocks: $w^{1118}$, w; elav-Gal4, w;UAS-DADm-DAAM::GFP (Matusek et al., 2008), w; UAS-CDAAM, Jupiter::GFP (Karpova et al., 2006), w;elavGal4,UAS-EB 1::GFP and UAS-Actin5C::GFP (Röper et al., 2005).

\section{Molecular biology and antibody production}

For Drosophila S2 cell expression of $\triangle \mathrm{DAD}-\mathrm{DAAM}$ we used a pAWF$\triangle$ DAD-DAAM destination clone created by standard methods. For bacterial protein expression the $\mathrm{DAD}, \mathrm{DAD}-\mathrm{CT}$ and $\mathrm{FH} 1 \mathrm{FH} 2^{1732 \mathrm{~A}}$ constructs were generated in the pGex2T vector by using standard cloning and in vitro mutagenesis methods. The DAD-CT ${ }^{\mathrm{R}-\mathrm{A}}$ mutant construct was created by gene synthesis (Sangon Biotech), and then cloned into pGex2T (Fig. S2F). Primers are listed in Table S1.

The DAAM antibody (Rb\#4938) was generated in rabbit after immunization with purified FH1FH2 produced in bacteria. The sera were collected, and the
IgG fraction was purified on ProteinG agarose beads. Specificity of the antibody was confirmed by western blot analysis (Fig. S3G).

\section{Cell cultures and immunohistochemistry}

Drosophila primary neuronal cells were obtained from stage 11 embryos as published in Sanchez-Soriano et al. (2010). S2 cells were grown in Schneider's medium supplemented with $10 \%$ heat-inactivated FBS.

For transfection of S2 cells, $2 \times 10^{6}$ cells were seeded in 6-well plates and then transfected with pAct5C- $\triangle \mathrm{DAD}-\mathrm{DAAM}:: 3 \times$ Flag construct using Effectene transfection reagent (Qiagen).

Cultured neurons were fixed at 6 hours in vitro (HIV) and stained as described in Matusek et al. (2008). The following primary antibodies were used: mouse anti- $\alpha$-tubulin $(1: 1000$, Sigma), rabbit anti-dDAAM (1:1000, Matusek et al., 2006), chicken anti-GFP (1:1000, Abcam). Actin was labeled with either Alexa-Fluor-488- or Alexa-Fluor-546-phalloidin (ThermoScientific).

For treatments with the microtubule-destabilizing drug nocodazole, neurons were plated onto coverslips coated with $0.5 \mathrm{mg} / \mathrm{ml}$ concanavalin A (Sigma) and kept at $22^{\circ} \mathrm{C}$ for $12 \mathrm{~h}$. After $12 \mathrm{~h}$, neurons were treated with either nocodazole (100 $\mu \mathrm{M}$; Sigma) or DMSO (both diluted in Schneider's medium) for $4 \mathrm{~h}$ at $22^{\circ} \mathrm{C}$. Control primary neurons were derived from white ${ }^{1118}$ embryos.

\section{Co-immunoprecipitation and western blots}

Co-immunoprecipitation was carried out as described previously (Gombos et al., 2015). Lysates of transfected $\mathrm{S} 2$ cells were incubated with rabbit antiEB1 antiserum, then samples were incubated with protein-A magnetic beads (Bio-Rad). Eluted proteins were analyzed by western blot using a standard procedure. Rabbit anti-EB1 (1:2000, gift from H. Ohkura), mouse anti-Flag (1:1000, M2, Sigma-Aldrich), mouse anti- $\alpha$-tubulin (1:10,000, DM1A, Sigma-Aldrich), anti-rabbit IgG-HRP (1:10,000, Jackson) and anti-mouse IgG-HRP (1:5000, Dako) were used for western blots.

\section{Protein expression and purification}

Drosophila DAAM constructs were expressed and purified as GST-tagged proteins as described (Barko et al., 2010). Actin was purified from rabbit skeletal muscle and labeled with Alexa-Fluor-568 carboxylic acid succinimidyl ester (Alexa568NHS, Invitrogen) (Kellogg et al., 1988; Spudich and Watt, 1971). $\mathrm{Mg}^{2+}$-ATP-actin filaments (F-actin) and phalloidin-stabilized F-actin were prepared as described (Tóth et al., 2016). Taxol-stabilized MTs were prepared from unmodified and/or HiLyte Fluor $^{\text {TM }}$ 488-labeled tubulin (Cytoskeleton, Inc.).

\section{MT-DAAM-binding assays \\ MT co-sedimentation}

MTs were prepared from tubulin protein (Cytoskeleton, Inc.) following the vendor's instructions. Purified GST-tagged $(4 \mu \mathrm{M})$ or GST-tagged dDAAM $(0.25-4 \mu \mathrm{M})$ fragments were incubated with taxol-stabilized MTs $(0.5 \mu \mathrm{M})$ for $30 \mathrm{~min}$ at room temperature in MT-binding buffer (MBB;10 mM HEPES pH 7.0, $1 \mathrm{mM} \mathrm{MgCl} 2,1 \mathrm{mM}$ EGTA, $1 \mathrm{mM}$ DTT, $20 \mu \mathrm{M}$ taxol, $0.5 \mathrm{mM}$ Thesit). Samples were centrifuged at $100,000 \mathrm{~g}$ for $25 \mathrm{~min}$. The pellets were washed in MBB then resuspended in SDS-PAGE sample buffer. Proteins in the supernatants and pellets were resolved by SDS-PAGE and stained by colloidal Coomassie Blue.

\section{GST pull-down assay}

GST- and GST-tagged DAAM fragments were expressed and purified from a small-volume culture $(2 \mathrm{ml})$ as described above. The proteins were not eluted from glutathione beads but, instead, were incubated with pre-assembled MTs $(0.5 \mu \mathrm{M})$ for $30 \mathrm{~min}$ in MBB. Beads were washed in MBB then the proteins were eluted and analyzed by SDS-PAGE and western blotting.

\section{MT stability assay}

The cold-induced depolymerization assay was carried out as described (Bartolini et al., 2008). Tubulin was polymerized in the presence of $4 \mu \mathrm{M}$ GST::FH1FH2 or GST::FH1FH2 proteins. After polymerization, matching aliquots of samples were centrifuged as described for MT co-sedimentation. The remaining samples were incubated on ice, then centrifuged at $4{ }^{\circ} \mathrm{C}$. Proteins 
in the supernatants and pellets were resolved by Coomassie Blue-stained SDSPAGE and the gels were quantified by using densitometry (ImageJ).

\section{MT/F-actin crosslinking assay}

Since both CDAAM and FH1-FH2 induces F-actin bundles, which sediment at very low speed, we adapted the sedimentation protocol used by Elie et al. (2015) to test the ability of DAAM to interact simultaneously with F-actin and MTs. Phalloidin-stabilized F-actin $(2 \mu \mathrm{M})$ and/or taxolstabilized MTs $(2 \mu \mathrm{M})$ were incubated with DAAM constructs $(9 \mu \mathrm{M})$ in Britton-Robinson buffer BRB-K (80 mM PIPES, pH 7.0, $2 \mathrm{mM} \mathrm{MgCl}_{2}$, $0.5 \mathrm{mM}$ EGTA, $50 \mathrm{mM} \mathrm{KCl}$ ) at room temperature for $40 \mathrm{~min}$. Samples were loaded onto a $30 \%$ sucrose cushion and centrifuged $\left(4000 \mathrm{~g}, 10 \mathrm{~min}, 25^{\circ} \mathrm{C}\right)$. Under these conditions individual polymers, as well as F-actin bundles do not sediment, only larger filament complexes (MT bundles, MT-F-actin copolymers) appear in the pellet (Elie et al., 2015). Pellets and supernatants were analyzed by SDS-PAGE. In control experiments based on high-speed centrifugation $\left(100,000 \mathrm{~g}, 20 \mathrm{~min}, 25^{\circ} \mathrm{C}\right)$ both F-actin and MTs appeared in the pellet, which confirms that both polymers exist under the applied experimental conditions, and the lack of sedimentation of the polymers is not due to depolymerization.

\section{Microscopy and image analysis}

Confocal images were captured either on a Zeiss Laser Scanning Microscope (LSM) 880 or on an Olympus FV1000 LSM. Images were restored using the Huygens Professional software (Scientific Volume Imaging). To visualize the F-actin and MT structures induced by DAAM, phalloidin-stabilized F-actin $(0.4 \mu \mathrm{M}$ containing $10 \%$ Alexa568NHStagged actin) and taxol-stabilized MTs $(0.4 \mu \mathrm{M}$ containing $10 \%$ HiLyte Fluor $^{\mathrm{TM}} 488$-tagged tubulin) were incubated with DAAM constructs $(1 \mu \mathrm{M})$ in BRB-K at room temperature for $30 \mathrm{~min}$. Samples were diluted in BRB$\mathrm{K}^{*}$ [BRB-K supplemented with $0.2 \%(\mathrm{w} / \mathrm{v})$ methylcellulose, $0.5 \%(\mathrm{w} / \mathrm{v})$ BSA, $50 \mathrm{mM}$ 1,4-diazabicyclo-[2,2,2]octane (DABCO) and $100 \mathrm{mM}$ DTT], applied onto poly-L-lysine-treated (Sigma Aldrich) coverslips and visualized by TIRF microscopy (Olympus IX 81). Microtubule-F-actin colocalization was quantified as described (Elie et al., 2015) (three to four independent experiments at each condition, 18-22 images). For nocodazole treatment in primary neurons, images were captured with a Nikon Eclipse 90i microscope equipped with a high-resolution CCD Camera (Retiga 3000). Images were processed using Image J software.

MT dynamics were analyzed from EB1::GFP time-lapse recordings by using TrackMate (v3.3.0). To allow particle detection and faithful tracking, the spatial and temporal resolution of the live recordings was set to $100 \mathrm{~nm} /$ voxel and $0.9 \mathrm{~s} /$ frame, respectively. Filopodial actin dynamics measurements were performed on 7-9 HIV neurons expressing Actin5C: GFP. Imaging of the neurons was performed in glass-bottomed Petri dishes (MatTek corporation) in growth medium. Filopodia with recognizable extension and retraction events were selected for further analysis.

\section{Statistics and figures}

Statistical analysis was carried out using Prism 5 (GraphPad Software Inc.) The D'Agostino-Pearson omnibus test was used to assess the normality of the data. Pairwise comparisons were made by using either Student's $t$-test or Mann-Whitney $U$-test according to the normality. ANOVA was used for multiple comparisons; $P<0.05$ was considered as statistically significant. Figures and drawings were created in Illustrator CS4 (Adobe).

\section{Acknowledgements}

We thank the Bloomington Stock Center, Hiro Ohkura (University of Edinburgh, Edinburgh, UK) and Andreas Prokop (University of Manchester, Manchester, UK) for fly stocks and reagents. We thank Éva Monostori (Biological Research Centre, Hungarian Academy of Sciences, Szeged, Hungary) for help with antibody production, and Anikó Berente, Edit Gyáni and Anna Rehák (Biological Research Centre, Hungarian Academy of Sciences, Szeged, Hungary) for technical assistance.

\section{Competing interests}

The authors declare no competing or financial interests.

\section{Author contributions}

Conceptualization: S.S., I.F., E.M., B.B., P.H., N.S.-S., J. Mihály; Methodology: S.S., I.F., J. Maléth; Formal analysis: S.S., I.F., K.T., E.M., A.V., B.B., J. Maléth, P.K.;
Investigation: S.S., I.F., K.T., E.M., A.V., B.B., J. Maléth, P.K., J. Mihály; Writing original draft: S.S., I.F., B.B., J. Mihály; Visualization: S.S., J. Maléth; Supervision: P.H., N.S.-S., J. Mihály.

\section{Funding}

This work was supported by the Országos Tudományos Kutatási Alapprogramok (Hungarian Science Foundation; OTKA) (grant numbers: K109330 to J.M., K109689 to B.B.), the Hungarian Brain Research Program (grant number: KTIA_NAP_13-22014-0007 to J.M.), the National Research, Development and Innovation Office (grant numbers: GINOP-2.3.2-15-2016-00001 and GINOP-2.3.2-15-2016-00032 to J.M.), the European Union and the State of Hungary, co-financed by the European Social Fund in the framework of TÁMOP 4.2.4.A/2-11-1-2012-0001 'National Excellence Program' and the New National Excellence Program of the Ministry of Human Capacities (to B.B.), the ÚNKP-16-4 New National Excellence Program of the Ministry of Human Capacities (to B.B.), the Biotechnology and Biological Sciences Research Council, BBSRC (grant number: BB/M007456/1 to N.S.-S), an MTA Postdoctoral Fellowship (to I.F.) and by the University of Liverpool and University of Szeged (Studentship to P.K.).

\section{Supplementary information}

Supplementary information available online at

http://jcs.biologists.org/lookup/doi/10.1242/jcs.203455.supplemental

\section{References}

Alberts, A. S. (2001). Identification of a carboxyl-terminal diaphanous-related formin homology protein autoregulatory domain. J. Biol. Chem. 276, 2824-2830.

Barko, S., Bugyi, B., Carlier, M.-F., Gombos, R., Matusek, T., Mihaly, J. and Nyitrai, M. (2010). Characterization of the biochemical properties and biological function of the formin homology domains of Drosophila DAAM. J. Biol. Chem. 285 13154-13169.

Bartolini, F. and Gundersen, G. G. (2010). Formins and microtubules. Biochim. Biophys. Acta 1803, 164-173.

Bartolini, F., Moseley, J. B., Schmoranzer, J., Cassimeris, L., Goode, B. L. and Gundersen, G. G. (2008). The formin mDia2 stabilizes microtubules independently of its actin nucleation activity. J. Cell Biol. 181, 523-536.

Bearce, E. A., Erdogan, B. and Lowery, L. A. (2015). TIPsy tour guides: how microtubule plus-end tracking proteins (+TIPs) facilitate axon guidance. Front. Cell Neurosci. 9, 241.

Bornschlogl, T., Romero, S., Vestergaard, C. L., Joanny, J.-F., Van Nhieu, G. T. and Bassereau, P. (2013). Filopodial retraction force is generated by cortical actin dynamics and controlled by reversible tethering at the tip. Proc. Natl. Acad. Sci. USA 110, 18928-18933.

Buck, K. B. and Zheng, J. Q. (2002). Growth cone turning induced by direct local modification of microtubule dynamics. J. Neurosci. 22, 9358-9367.

Burnette, D. T., Schaefer, A. W., Ji, L., Danuser, G. and Forscher, P. (2007) Filopodial actin bundles are not necessary for microtubule advance into the peripheral domain of Aplysia neuronal growth cones. Nat. Cell Biol. 9, 1360-1369. Cammarata, G. M., Bearce, E. A. and Lowery, L. A. (2016). Cytoskeletal socia networking in the growth cone: How +TIPs mediate microtubule-actin crosslinking to drive axon outgrowth and guidance. Cytoskeleton 73, 461-476.

Cheng, L., Zhang, J., Ahmad, S., Rozier, L., Yu, H., Deng, H. and Mao, Y. (2011). Aurora B regulates formin mDia3 in achieving metaphase chromosome alignment. Dev. Cell 20, 342-352.

Chesarone, M. A., DuPage, A. G. and Goode, B. L. (2010). Unleashing formins to remodel the actin and microtubule cytoskeletons. Nat. Rev. Mol. Cell Biol. 11, 62-74

Coles, C. H. and Bradke, F. (2015). Coordinating neuronal actin-microtubule dynamics. Curr. Biol. 25, R677-R691.

Coué, M., Brenner, S. L., Spector, I. and Korn, E. D. (1987). Inhibition of actin polymerization by latrunculin A. FEBS Lett. 213, 316-318.

Dahlgaard, K., Raposo, A. A. S. F., Niccoli, T. and St Johnston, D. (2007). Capu and Spire assemble a cytoplasmic actin mesh that maintains microtubule organization in the Drosophila oocyte. Dev. Cell 13, 539-553.

Dent, E. W. and Gertler, F. B. (2003). Cytoskeletal dynamics and transport in growth cone motility and axon guidance. Neuron $\mathbf{4 0}, 209-227$.

Dent, E. W. and Kalil, K. (2001). Axon branching requires interactions between dynamic microtubules and actin filaments. J. Neurosci. 21, 9757-9769.

Elie, A., Prezel, E., Guérin, C., Denarier, E., Ramirez-Rios, S., Serre, L., Andrieux, A., Fourest-Lieuvin, A., Blanchoin, L. and Arnal, I. (2015). Tau coorganizes dynamic microtubule and actin networks. Sci. Rep. 5, 9964.

Forscher, P. and Smith, S. J. (1988). Actions of cytochalasins on the organization of actin filaments and microtubules in a neuronal growth cone. J. Cell Biol. 107, 1505-1516.

Gaillard, J., Ramabhadran, V., Neumanne, E., Gurel, P., Blanchoin, L., Vantard, M. and Higgs, H. N. (2011). Differential interactions of the formins INF2, mDia1 and $\mathrm{mDia} 2$ with microtubules. Mol. Biol. Cell 22, 4575-4587.

Gasteier, J. E., Schroeder, S., Muranyi, W., Madrid, R., Benichou, S. and Fackler, O. T. (2005). FHOD1 coordinates actin filament and microtubule alignment to mediate cell elongation. Exp. Cell Res. 306, 192-202. 
Gombos, R., Migh, E., Antal, O., Mukherjee, A., Jenny, A. and Mihaly, J. (2015) The formin DAAM functions as molecular effector of the planar cell polarity pathway during axonal development in Drosophila. J. Neurosci. 35, 10154-10167.

Gonçalves-Pimentel, C., Gombos, R., Mihály, J., Sánchez-Soriano, N. and Prokop, A. (2011). Dissecting regulatory networks of filopodia formation in a Drosophila growth cone model. PLoS ONE 6, e18340.

Gould, C. J., Maiti, S., Michelot, A., Graziano, B. R., Blanchoin, L. and Goode, B. L. (2011). The formin DAD domain plays dual roles in autoinhibition and actin nucleation. Curr. Biol. 21, 384-390.

Henty-Ridilla, J. L., Rankova, A., Eskin, J. A., Kenny, K. and Goode, B. L. (2016) Accelerated actin filament polymerization from microtubule plus ends. Science 352, 1004-1009.

Higashida, C., Miyoshi, T., Fujita, A., Oceguera-Yanez, F., Monypenny, J., Andou, Y., Narumiya, S. and Watanabe, N. (2004). Actin polymerization-driven molecular movement of mDia1 in living cells. Science 303, 2007-2010.

Jaiswal, R., Breitsprecher, D., Collins, A., Corrêa, I. R., Jr, Xu, M.-Q. and Goode, B. L. (2013a). The formin Daam1 and fascin directly collaborate to promote filopodia formation. Curr. Biol. 23, 1373-1379.

Jaiswal, R., Stepanik, V., Rankova, A., Molinar, O., Goode, B. L. and McCartney, B. M. (2013b). Drosophila homologues of adenomatous polyposis coli (APC) and the formin diaphanous collaborate by a conserved mechanism to stimulate actin filament assembly. J. Biol. Chem. 288, 13897-13905.

Jiang, K., Toedt, G., Montenegro Gouveia, S., Davey, N. E., Hua, S., van der Vaart, B., Grigoriev, I., Larsen, J., Pedersen, L. B., Bezstarosti, K. et al. (2012). A Proteome-wide screen for mammalian SxIP motif-containing microtubule plusend tracking proteins. Curr. Biol. 22, 1800-1807.

Karpova, N., Bobinnec, Y., Fouix, S., Huitorel, P. and Debec, A. (2006). Jupiter, a new Drosophila protein associated with microtubules. Cell Motil. Cytoskeleton 63 , 301-312.

Kellogg, D. R., Mitchison, T. J. and Alberts, B. M. (1988). Behaviour of microtubules and actin filaments in living Drosophila embryos. Developmen 103, 675-686.

Komarova, Y., De Groot, C. O., Grigoriev, I., Gouveia, S. M., Munteanu, E. L., Schober, J. M., Honnappa, S., Buey, R. M., Hoogenraad, C. C., Dogterom, M. et al. (2009). Mammalian end binding proteins control persistent microtubule growth. J. Cell Biol. 184, 691-706.

Kovar, D. R. and Pollard, T. D. (2004). Insertional assembly of actin filament barbed ends in association with formins produces piconewton forces. Proc. Natl. Acad. Sci. USA 101, 14725-14730.

Lewkowicz, E., Herit, F., Le Clainche, C., Bourdoncle, P., Perez, F. and Niedergang, F. (2008). The microtubule-binding protein CLIP-170 coordinates mDia1 and actin reorganization during CR3-mediated phagocytosis. J. Cell Biol. 183, 1287-1298.

Li, F. and Higgs, H. N. (2003). The mouse Formin mDia1 is a potent actin nucleation factor regulated by autoinhibition. Curr. Biol. 13, 1335-1340.

Li, F. and Higgs, H. N. (2005). Dissecting requirements for auto-inhibition of actin nucleation by the formin, mDia1. J. Biol. Chem. 280, 6986-6992.

Liu, W., Sato, A., Khadka, D., Bharti, R., Diaz, H., Runnels, L. W. and Habas, R. (2008). Mechanism of activation of the Formin protein Daam1. Proc. Natl. Acad. Sci. USA 105, 210-215.

Lowery, L. A. and Van Vactor, D. (2009). The trip of the tip: understanding the growth cone machinery. Nat. Rev. Mol. Cell Biol. 10, 332-343.

Marx, A., Godinez, W. J., Tsimashchuk, V., Bankhead, P., Rohr, K. and Engel, U. (2013). Xenopus cytoplasmic linker-associated protein 1 (XCLASP1) promotes axon elongation and advance of pioneer microtubules. Mol. Biol. Cell 24, 1544-1558.

Matusek, T., Djiane, A., Jankovics, F., Brunner, D., Mlodzik, M. and Mihaly, J. (2006). The Drosophila formin DAAM regulates the tracheal cuticle pattern through organizing the actin cytoskeleton. Development 133, 957-966.

Matusek, T., Gombos, R., Szecsenyi, A., Sanchez-Soriano, N., Czibula, A., Pataki, C., Gedai, A., Prokop, A., Rasko, I. and Mihaly, J. (2008). Formin proteins of the DAAM subfamily play a role during axon growth. J. Neurosci. 28 $13310-13319$.

Meijering, E., Jacob, M., Sarria, J.-C. F., Steiner, P., Hirling, H. and Unser, M. (2004). Design and validation of a tool for neurite tracing and analysis in fluorescence microscopy images. Cytometry 58A, 167-176.

Morrison, E. E., Moncur, P. M. and Askham, J. M. (2002). EB1 identifies sites of microtubule polymerisation during neurite development. Brain Res. Mol. Brain Res. 98, 145-152.

Okada, K., Bartolini, F., Deaconescu, A. M., Moseley, J. B., Dogic, Z., Grigorieff, N., Gundersen, G. G. and Goode, B. L. (2010). Adenomatous polyposis col protein nucleates actin assembly and synergizes with the formin mDia1. J. Cell Biol. 189, 1087-1096.

Otomo, T., Otomo, C., Tomchick, D. R., Machius, M. and Rosen, M. K. (2005) Structural basis of Rho GTPase-mediated activation of the formin mDia1. Mol. Cell $18,273-281$
Röper, K., Mao, Y. and Brown, N. H. (2005). Contribution of sequence variation in Drosophila actins to their incorporation into actin-based structures in vivo. J. Cell Sci. 118, 3937-3948.

Rosales-Nieves, A. E., Johndrow, J. E., Keller, L. C., Magie, C. R., Pinto-Santini, D. M. and Parkhurst, S. M. (2006). Coordination of microtubule and microfilament dynamics by Drosophila Rho1, Spire and Cappuccino. Nat. Cell Biol. 8, 367-376.

Rose, R., Weyand, M., Lammers, M., Ishizaki, T., Ahmadian, M. R. and Wittinghofer, A. (2005). Structural and mechanistic insights into the interaction between Rho and mammalian Dia. Nature 435, 513-518.

Roth-Johnson, E. A., Vizcarra, C. L., Bois, J. S. and Quinlan, M. E. (2014) Interaction between microtubules and the Drosophila formin Cappuccino and its effect on actin assembly. J. Biol. Chem. 289, 4395-4404

Sagot, I., Rodal, A. A., Moseley, J., Goode, B. L. and Pellman, D. (2002). An actin nucleation mechanism mediated by Bni1 and profilin. Nat. Cell Biol. 4, 626-631.

Sanchez-Soriano, N., Goncalves-Pimentel, C., Beaven, R., Haessler, U. Ofner-Ziegenfuss, L., Ballestrem, C. and Prokop, A. (2010). Drosophila growth cones: a genetically tractable platform for the analysis of axonal growth dynamics. Dev. Neurobiol. 70, 58-71.

Schaefer, A. W., Kabir, N. and Forscher, P. (2002). Filopodia and actin arcs guide the assembly and transport of two populations of microtubules with unique dynamic parameters in neuronal growth cones. J. Cell Biol. 158, 139-152.

Schaefer, A. W., Schoonderwoert, V. T. G., Ji, L., Mederios, N., Danuser, G. and Forscher, P. (2008). Coordination of actin filament and microtubule dynamics during neurite outgrowth. Dev. Cell 15, 146-162.

Schönichen, A. and Geyer, M. (2010). Fifteen formins for an actin filament: a molecular view on the regulation of human formins. Biochim. Biophys. Acta 1803 152-163.

Shaye, D. D. and Greenwald, I. (2015). The disease-associated formin INF2/EXC-6 organizes lumen and cell outgrowth during tubulogenesis by regulating F-actin and microtubule cytoskeletons. Dev. Cell 32, 743-755.

Spudich, J. A. and Watt, S. (1971). The regulation of rabbit skeletal muscle contraction. I. Biochemical studies of the interaction of the tropomyosin-troponin complex with actin and the proteolytic fragments of myosin. J. Biol. Chem. 246 4866-4871.

Stepanova, T., Slemmer, J., Hoogenraad, C. C., Lansbergen, G., Dortland, B., De Zeeuw, C. I., Grosveld, F., van Cappellen, G., Akhmanova, A. and Galjart N. (2003). Visualization of microtubule growth in cultured neurons via the use of EB3-GFP (end-binding protein 3-green fluorescent protein). J. Neurosci. 23 2655-2664.

Tinevez, J. Y., Perry, N., Schindelin, J., Hoopes, G. M., Reynolds, G. D., Laplantine, E., Bednarek, S. Y., Shorte, S. L. and Eliceiri, K. W. (2017). TrackMate: an open and extensible platform for single-particle tracking. Methods 115, 80-90.

Tóth, M. A., Majoros, A. K., Vig, A. T., Migh, E., Nyitrai, M., Mihály, J. and Bugyi, B. (2016). Biochemical activities of the Wiskott-Aldrich syndrome homology region 2 domains of sarcomere length short (SALS) protein. J. Biol. Chem. 291 $667-680$

Vizcarra, C. L., Bor, B. and Quinlan, M. E. (2014). The role of formin tails in actin nucleation, processive elongation, and filament bundling. J. Biol. Chem. 289 30602-30613

Watanabe, N. and Higashida, C. (2004). Formins: processive cappers of growing actin filaments. Exp. Cell Res. 301, 16-22.

Waterman-Storer, C. M. and Salmon, E. D. (1998). How microtubules get fluorescent speckles. Biophys. J. 75, 2059-2069.

Wen, Y., Eng, C. H., Schmoranzer, J., Cabrera-Poch, N., Morris, E. J. S., Chen, M., Wallar, B. J., Alberts, A. S. and Gundersen, G. G. (2004). EB1 and APC bind to $\mathrm{mDia}$ to stabilize microtubules downstream of Rho and promote cell migration. Nat. Cell Biol. 6, 820-830.

Wu, Y., Eghbali, M., Ou, J., Lu, R., Toro, L. and Stefani, E. (2010). Quantitative determination of spatial protein-protein correlations in fluorescence confocal microscopy. Biophys. J. 98, 493-504

Yarmola, E. G., Somasundaram, T., Boring, T. A., Spector, I. and Bubb, M. R. (2000). Actin-latrunculin A structure and function. Differential modulation of actinbinding protein function by latrunculin A. J. Biol. Chem. 275, 28120-28127.

Young, K. G. and Copeland, J. W. (2010). Formins in cell signaling. Biochim. Biophys. Acta 1803, 183-190.

Young, K. G., Thurston, S. F., Copeland, S., Smallwood, C. and Copeland, J. W. (2008). INF1 is a novel microtubule-associated formin. Mol. Biol. Cell 19, 5168-5180.

Zhou, F.-Q. and Cohan, C. S. (2004). How actin filaments and microtubules steer growth cones to their targets. J. Neurobiol. 58, 84-91.

Zhou, F., Leder, P. and Martin, S. S. (2006). Formin-1 protein associates with microtubules through a peptide domain encoded by exon-2. Exp. Cell Res. 312 1119-1126.

Zinchuk, V., Wu, Y., Grossenbacher-Zinchuk, O. and Stefani, E. (2011). Quantifying spatial correlations of fluorescent markers using enhanced background reduction with protein proximity index and correlation coefficien estimations. Nat. Protoc. 6, 1554-1567. 